\title{
2 Analysis of the Densification Process
}

Solving problems associated with the densification process requires complete knowledge about the pressing process of wood biomass itself. This means that we must understand the distribution of pressing forces; parameters affecting the pressing process and their related interactions; ideal material for pressing and theirstructureand properties; dependencies between material properties and densification parameters; as well as existing pressing technologies. We know that wood, in terms of recovering energy through densification, must be prepared in a way that is advantageous for pressing. Wood biomass intended for pressing must be disintegrated and, in some cases, sorted to obtain a homogeneous fraction size. It is also necessary to obtain ideal water content either through drying (commonly used) or humidification (spraying, steaming). Transforming wood biomass into a usable biofuel commonly utilizes wood chips or saw dust as a raw input material. With these, it is necessary to understand their mechanical and chemical properties, particle geometry, force interactions between individual particles, and the effect of these "material" properties on the pressing process. Thus, these parameters must be considered to predict and ensure the final quality of the briquette product. Standards exist which determine the quality of the briquette. Understanding the quid lines within these standards allows for the solution to many problems that originate from the design of the pressing machine.

\subsection{Particulate Matter from Wood Biomass}

Particulate matter from wood biomass are composed of contacting solid particles (solid phase) and liquid/gas phase. The liquid/gas portion fills the pores between the solid particles, known as the external pores. Internal pores exist within the particles themselves. If the solid particles are sufficiently porous, the surface (open) pores and internal (closed) pores are known. The quantity of liquid/gas within the external pores can vary. Sometimes it is in the form of absorbed water on the surface pores of the solid particles, and it can penetrate into the internal pores of the particles themselves, which substantially changes their geometric and physical properties. Surface pores increase the surface area of particles while internal pores effect the density or strength of the solid particles.

The relationship between the size of the surface and properties of the solid particles is essentially that, when enlarging the size of the surface, the amount of atoms and thus the overall size of the interface between phases also increases. Efforts in increasing the number of binding imbalances between atoms commonly manifests in a large spectrum of varying surface interactions, which are mainly in absorption and catalytic effects that result in significant change to the colloidal and hydraulic properties.

\section{(cc) BY-NC-ND 2014 Peter Križan}

This work is licensed under the Creative Commons Attribution-NonCommercial-NoDerivs 3.0 License. 


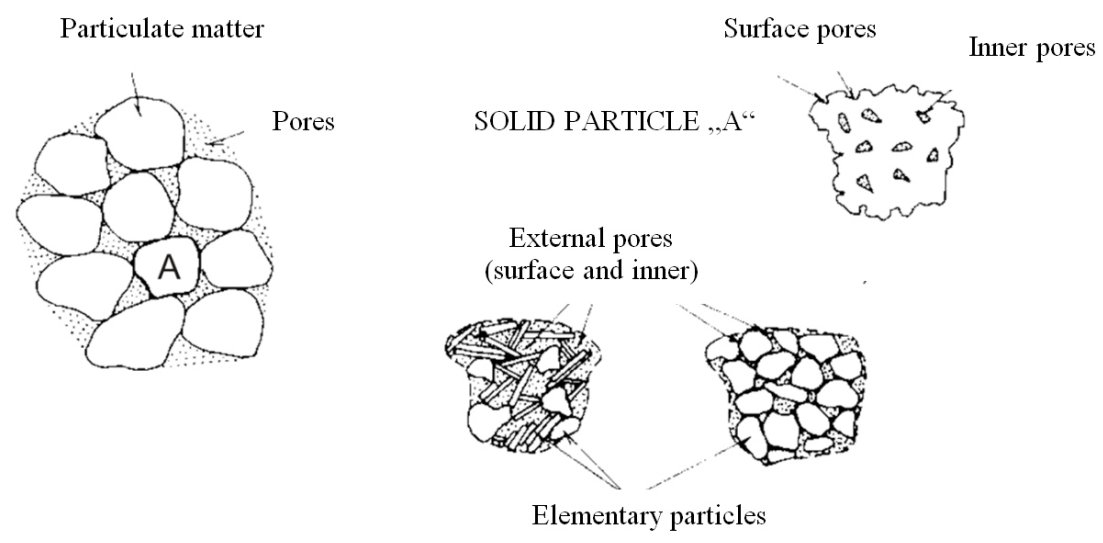

Figure 2.1: Solid particles “A” in particulate matter (Jindra, 1984).

Increasing the surface of the solid substance has the natural effect of increasing its reactivity - as is truein homogeneous and heterogeneous reactions. Chemical reactivity is essentially proportional to the surface size. Exceptions to this are situations when the surface pores are coated with an inert or non-reactive substance. However, in cases where the particle size is reduced by means of mechanical-chemical methods (shredding, for example) the exposed internal surfaces also increase the reactivity. There are many examples which document the mutual relationship between surface size and properties of solid particulate matter. However, it is necessary to remark that the change in physical-chemical properties of solid particles is related to only significant changes in surface size. Nevertheless, it is obvious that even small changes in particle size fundamentally affect the spectrum of application for these substances. Therefore, the size of the surface affects most of the substance properties (Feda, 1977). The relationship between surface size and physical-chemical properties of the solid particles resides in the fact that increasing the size of the surface increases the number of atoms that compose the interface between phases. For individual particles, the amount of atoms in the interface between phases increases relatively, which increases the total number of atoms in the particles system.

Solid particles in particulate matter contact each other. This contact restricts free movement between individual solid particles and thus affects the particulate matter strength and stiffness. The particle degrees of freedom in wood chips change with respect to the magnitude of stress throughout the deformation process. Increasing stress reduces the degrees of freedom until the magnitude of stress reaches the yield stress of the particle (wood chip), resulting in the disintegration and subsequent increase in movement freedom. 


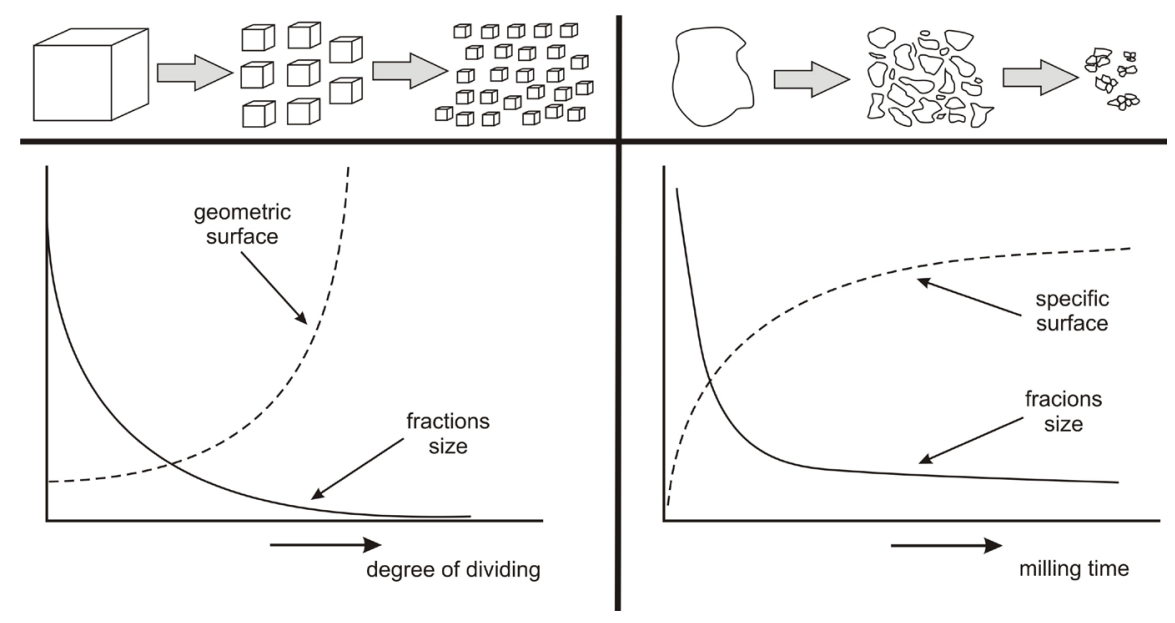

Figure 2.2: Difference between changing surface size of abstract and real solid bodies (Jesenák, 2008).

This property is exploited in the pressing process of solid particles, in which the action of pressure and heat densify solid particles into a compact whole. However, when pressing, stress must not exceed the yield strength of the solid particles. The goal is to minimize the pores occupying space between solid particles, contribute to stronger interactions between individual particles, and thus obtain a higher particle density briquette.

\subsection{Structure of Wood Biomass Particulate Matter}

The mechanical properties of particulate matter depend also upon their arrangements. If we are considering the arrangement of particles, we are talking about the structure or texture of the particulate matter. The structure (internal) of particulate matter defines the character and geometric arrangement of the solid particles and the behaviour of interactions between them. This behaviour depends on the number of structural bonds, which is a result of the size, shape, roughness, and strength of the solid particle. All of these factors influence the conceptual structure of the particulate matter and its heterogeneity (alternating surfaces of fine and course particles; particles of varying composition, shape, orientation), known as the texture or sometimes known as the macrostructure. It is important to differentiate between the structure of substances composed from particles of varying size (substances with varying degrees of polydispersion) and substances with equal polydispersion but with varying absolute size of particle. Fig. 2.3 describes examples of particulate matter composition (structure). In general, the most complex structure occurs when varying particulate matters are mixed together. 


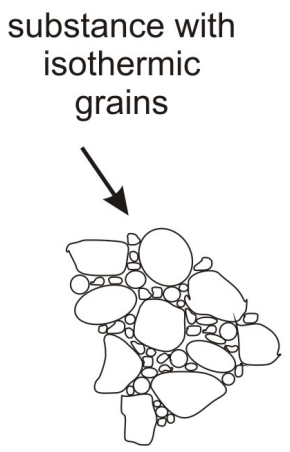

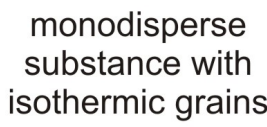

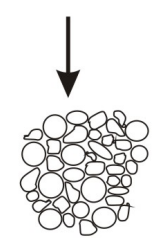

flocculated

structure

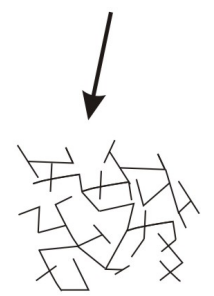

Figure 2.3: Examples of particulate matter structure (Jesenák, 2008).

In terms of pressing, it is very important to know the composition of the particulate matter, its structure, and the size of particles. The size and structure significantly affect the bonding mechanisms and the strength of these interactions upon pressing. These properties affect the continuity of the densification process and quality of the briquette product.

Factors that restrict the structure of the particulate matter can be divided into three groups: character of the solid particles, geometric arrangement of the particles, and the behaviour between them. In the first group belongs, foremost, the individual and group characteristics of the solid particles: the strength, composition, and structure of the solid particle; and the size, shape, and roughness. The most widely used characteristic of this group is the granularity curve - diagram of the particle phase composition (Fig. 2.4). It is possible to change the phase composition before the pressing process. The amount of water can be reduced, to a degree, by drying. The smaller the particle, the higher the effectiveness when drying.

However, this statement doesn't apply over the complete range of particle sizes due to compliance with safety factors when drying so as to prevent combustion of dust particles. The goal is obviously not to obtain completely dry particulate matter (complete removal of water). If completely dry particulate matter were pressed, we would obtain sintered particles, which are unacceptable.

Unlike the described properties, parameters such as structure and state variables form the second and third group of factors. Examining these factors requires following the substance state, which necessitates using an undisturbed sample. The second group of factors defines the geometric arrangement of the solid phase. The simplest of them is the average volumetric mass from which we can derive porosity. If solid particles create a cluster of elementary particles, then it is important to describe the geometric arrangement of the clusters.

The third group of structural characteristics includes the bonds between solid particles. For particulate substances, it is possible to differentiate two types of bonds: 


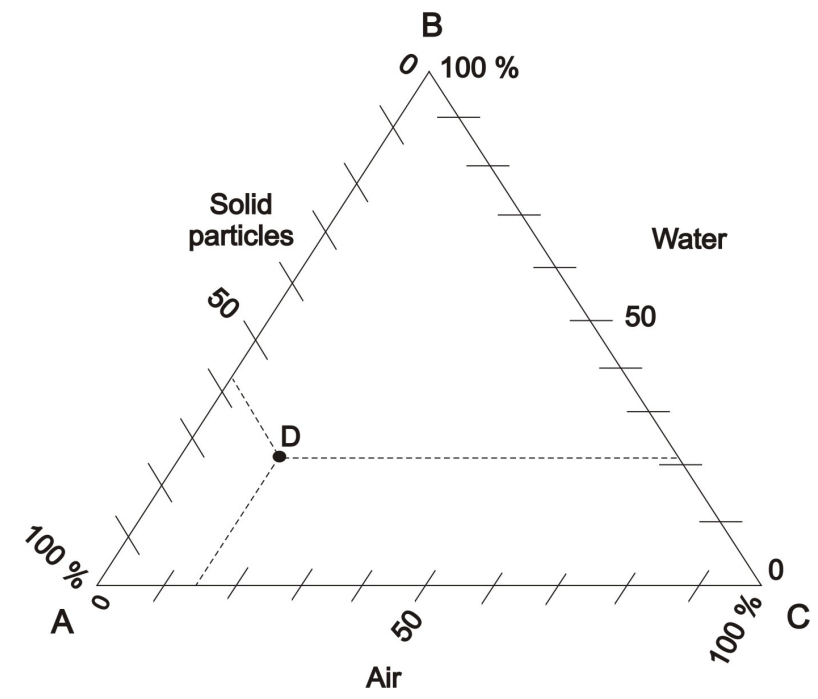

Figure 2.4: Triangle diagram representing the phase compositions (Feda, 1977).

bonds effected by external loading (including weight), which are referred to as frictional bonds, and bonds created from internal stress, referred to as cohesive bonds. These occur as a result of varying bonding mechanisms that affect the mechanical properties as well as the pressing process itself. In Section 2.4, we describe them in more detail. The theoretically calculated tensile strength of particles $\sigma_{t}$ in relation to the size of the particle $x$ from the initial substance for varying bonding mechanisms can be seen in Fig. 2.5. The dashed line parallel to the $\mathrm{x}$-axis divides the diagram into areas I and II. Area I represents a briquette subject to high pressure. In this area, the particles of the briquette are in surface contact (Jindra, 1984). Area II represents the pressing process where bridges are applied around the contacts between particles (Jindra, 1984).

The diagonal lines divide the diagram into regions, which define the bonding mechanisms. From this diagram, it is also clear that disintegrating wood biomass into very small particles is good practice before pressing so that we obtain the best mutual bonding between material particles.

\subsection{Predominant Mechanisms of Internal (force) Bonds}

Related contact phases in the particulate substance create force bonds between particles based on various mechanisms. These bonds affect all mechanical properties of the particulate matter. Even though detailed research about the effects of individual bonding mechanisms exist, only a few of these are suitable for the mathematical 


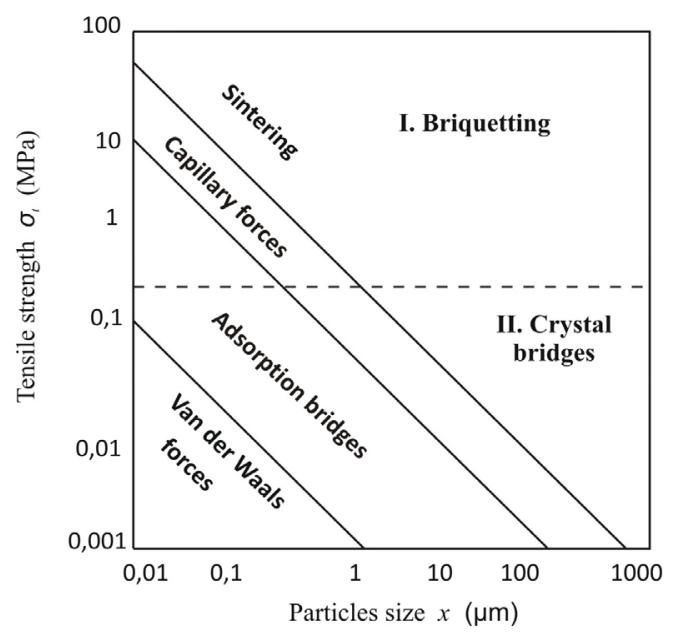

Figure 2.5: Theoretical strength of the bonded substance particles in relation to the size of particle before pressing (Jindra, 1984; Medek, 1991).

description of the particulate system. The particle contact strength is a collection of elementary bonds on the surface of the actual contact. In real particulate substances, all types of bonding mechanisms practically act together. The known theoretical approach in calculating the adhesion forces on a part of a system apply to roughness. The Jenkins test, a shear test, is used to experimentally prove the calculations. The tensile strength of adhesion can be specified from the characteristic values obtained for bulk material, i.e. many points of contacting particles characterised by the coordinate $\mathrm{K}$ and roughness of the contact surface. Emerging bonds between particles influence several factors, mostly the saturation of liquid (amount of liquid component on the surface of the particle and between particles).

Under current definitions, we can distinguish between:

- dry particulate substance - a substance that does not contain any liquid phase; only a solid consisting of particles with air/gas filling the spaces between them. Saturation S $=0$ (Fig. 2.6a).

- damp particulate substance- a substance which consists of a liquid phase together with the solid phase, where some liquid occupies the spaces between particles. Saturation $0<\mathrm{S}<1$ (Fig. 2.6b, c, d).

- paste - a particulate substance which consists of only a liquid phase comprised of particles whose pores are completely filled with liquid. Saturation $S=1$ (Fig. 2.6e). 


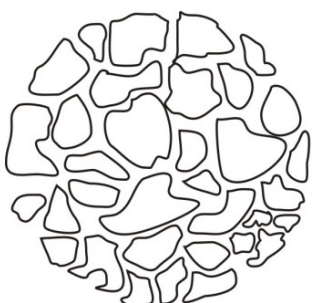

(a)

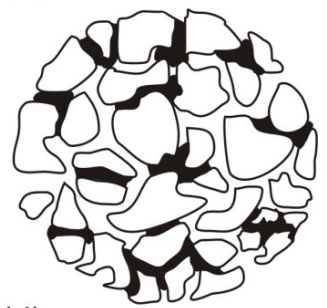

(d)

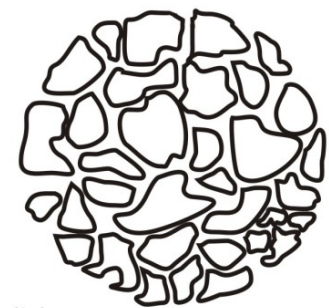

(b)

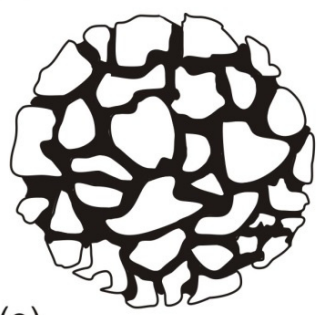

(e)

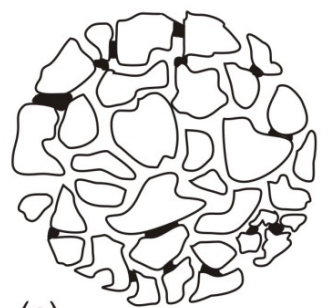

(c)

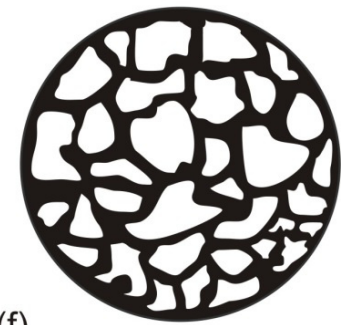

(f)

Figure 2.6: Schematic representation of varying degrees of saturation between particles (Pietsch, 1991).(a)no saturation - dry; (b) absorption layers; (c) liquid bridges (pendular state); (d) transition state (funicular state); (e) completely saturated state (liquid state); (f) droplet.

According to Pietsch(1991),we know five main groups of bonding mechanisms and several subgroups:

1. Bridges of solid character- bonds that occur between the contacts of the particles created by bridges in the solid phase. Their occurrence results in:

- sintered particles (diffusion of atoms and molecules at higher temperatures);

- partial melting of the particle surface (melting adhesion - cold welding);

- chemical reaction between particles (usually activated by moisture);

- $\quad$ hardening of the binding between particles (usually activated by moisture);

- recrystallization of the solid phase from the liquid component;

- drying (recrystallization of soluble components and the elimination of non-liquid particles).

2. Adhesion-cohesion forces - bonds created by adhesion in the interfacing surfaces and action of internal cohesive forces caused by:

- use of high-viscosity adhesives (particle bonding with a high-viscosity adhesive),

- formation of absorption layers $/<3 \eta \mathrm{m} /$ (small particles attracting surrounding atoms and molecules creating an absorbing layer.

3. Surface stress and capillary pressure- interactions with the most common bonding mechanism between particles of moist materials being:

- liquid bridges (created in materials with a higher liquid content in the contact area (water, capillary condensation), 
- capillary pressure (if the pore volume between particles in the disperse system is filled with water, capillary pressure creates a bond between the pores and surface of the particles).

4. Attractive forces between particles-even in cases where bridges of some substance do not occur between particles, a force bond caused by molecular interactions create fields in the particles. The particles themselves can emit these fields which we categorize as:

- molecular forces (Van der Waals force, valence forces - free chemical bonds hydrogen bonds),

- electrostatic forces,

- magnetic forces.

5. Shape bonds-particles in the shape of fibres, flakes, petals, or particles with irregular surfaces that attach/tangle with each other when pressed.

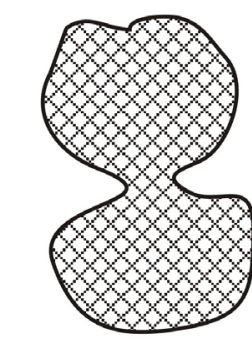

- sinter bridges;

- partial melting;

- crystallization of soluble substances;

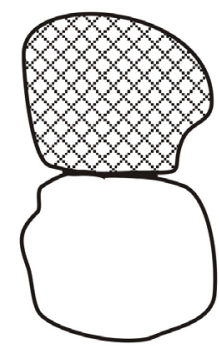

- chemical reaction between particles;

- hardening binders;

- highly viscous binders;

- adsorption layers;

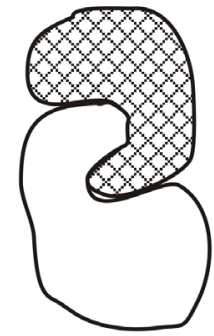

- interlocking;

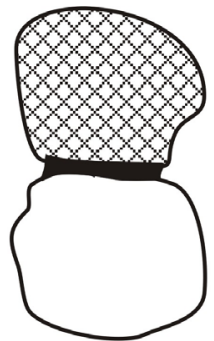

- liquid bridges;

- hardening binders; - crystallization of dissolved substances;

- deposition of suspended particles;

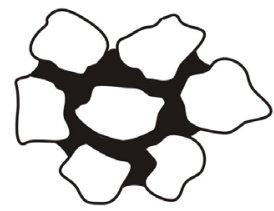

- chemical reaction between particles; - hardening binders; - highly viscous binders; - capillary forces;

- electrostatic forces;

- magnetic forces;

Figure 2.7: Schematic (illustration) representing bonding mechanisms (Pietsch, 1991). 
Bonding forces caused by the mechanisms discussed above can be considered internal cohesive forces acting between particles. These forces ensure a bond between particles even after we release external forces acting on the particles. Normal compression and friction forces occur in the areas loaded by external forces.

\subsection{Properties of Disintegrated Wood Biomass}

When cutting, disintegrating, or processing wood, chips and saw dust are a resulting by-product. Their shape, size, and quantity are dependent on the physical-mechanical properties of wood as well as the shape, size, and sharpness of the cutting tool or technology used to process the wood. We know that the process of wood disintegration is important with respect to the size of input fractions when pressing. Therefore, it is necessary to know the properties of disintegrated wood.

Chips - wood particles caused by cutting, disintegration, or machining of wood. Depending on the method of processing, these particles are also known as splinters, shavings, dust, or fibres and can be unified under a common term - disintegrated wood mass (DWM). DWM characteristically does not occupy the whole space of its bulk mass; air spaces exist between the solid particles. This reality puts DWM into the category of dispersion - a disproportionate system composed of two phases. Another typical property of DWM is that when each individual wood particle shifts, the overall external shape of the bulk changes as well. These properties specify DWM as a loose bulk material.

Amongst the basic descriptors characterising the physical and physical-chemical properties of bulk DWM are:

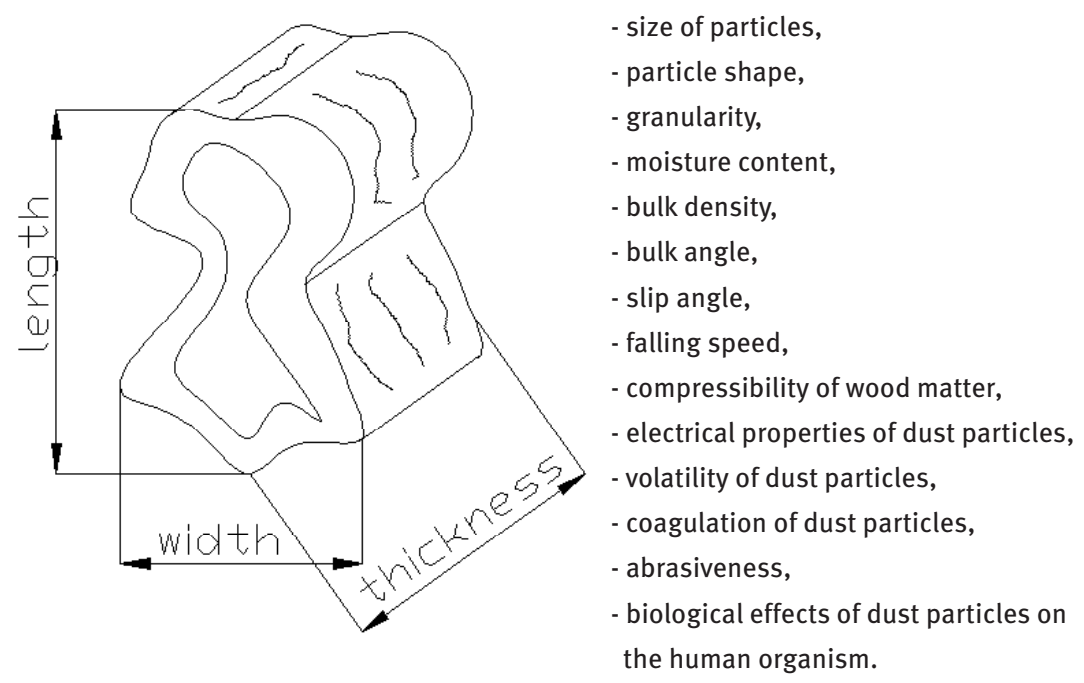

Figure 2.8: General dimensions of a DWM particle (Hejma\& et al., 1981). 
All of the above-mentioned properties describe and characterize DWM; however, not all of them are important in terms of pressing. The main parameters (properties) that characterize DWM are particle size and shape, granularity (granular composition), moisture, bulk density, and particle compressibility. In the next part of this monograph, we will dedicate more attention to these properties, since they directly affect the pressing process of wood materials.

\subsubsection{Particle Size of DWM}

The size of DWM particles is one of the most basic properties. It affects the physical properties of the bulk DWM such as bulk density, granular composition, slip angle, and pour angle. It also affects the movement properties of the particle in various transport systems, vacuum systems, and drying and separation conditions.

Particle dimensions are defined as the dimensions of the right angle parallelogram described by a given particle, where:

- length is the largest dimension of the particle,

- thickness is the smallest dimension of the particle,

- width is the third dimension of the particle.

Particle size defines the largest dimension of the right angle parallelogram around a particle (length of particle).

DWM particles resulting in wood cutting, disintegration, or wood-working differ considerably in size. A homogeneous fraction size can be achieved only in disintegrating machines or through sieving/screening the raw material. The maximum size of the particle is dependent on the type of disintegrating machine, size of the disintegrating wedges, or screen size following the disintegrating chamber. The maximum and minimum particle dimension from wood-working can be seen in Table 2.1. In the disintegration process, the maximum and minimum particle dimensions depend on the size of the screen.

\subsubsection{Particle Shape of DWM}

As was mentioned before, the geometric shape of wood particles depends on the wood-working process, and each result in very diverse dimensions. Nevertheless, we can attempt to divide each type of bulk material into groups:

- isometric particles - particles which have approximately the same dimension in all three directions (fine chip fractions, fine dust fractions)

- flat particles (laminar) - length and width are significantly larger than the thickness (splinters, shavings, flakes).

- fibre particles - particles with significant elongation in one direction (fibre, medium thickness dust particle fractions). 
Table 2.1: Size of DWM particles (Dzurenda, 2002).

\begin{tabular}{lll}
\hline DWM particle method & $\begin{array}{l}\text { Particle Size } \\
\text { minimum }(\mathrm{mm})\end{array}$ & maximum (mm) \\
\hline Chips (wood chipper) & 2.5 & 40 \\
Splinter (wood disintegrating machine) & 0.16 & 6.5 \\
Wet sawdust (sawdust from wood ban saw) & 0.064 & 4.8 \\
Dry sawdust (longitudinal circular saw) & 0.027 & 3.5 \\
Dry wood fibres & 0.5 & 4 \\
Wood dust (belt sanding) & 0.001 & 0.5 \\
\hline
\end{tabular}

Depending on the technological properties of DWM and on other properties, an effort is made to characterize bulk particles by a single dimension. Depending on the processing method of DWM, such a dimension can be defined as follows:

- equivalent particle diameter $a_{A}$ - diameter of the circle $a_{A}$, whose area $S_{p}$ is the same size as the projected real particle areain the direction of its thickness $S_{\check{c}}$ (Fig. 2.9).

$$
a_{A}=\sqrt{\frac{4 * S_{p}}{\pi}}(\mathrm{mm})
$$
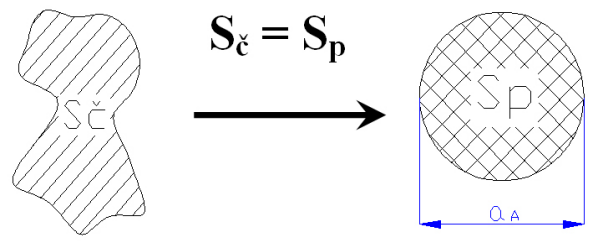

Figure 2.9: Equivalent particle size according to the projection of $a_{A}$ (Dzurenda, 2002).

- equivalent particle diameter $a_{V}$ - is a diameter of the sphere whose volume is $V g$ is equivalent to the real particle $V_{\breve{c}}$.

$$
a_{V}=\sqrt[3]{\frac{6^{*} V_{g}}{\pi}}(\mathrm{mm})
$$




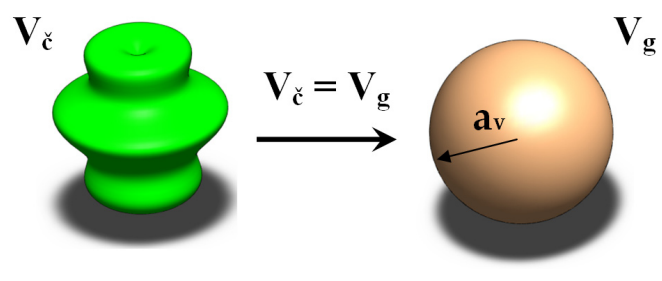

Figure 2.10: Equivalent particle size with respect to volume $a_{v}$ (Dzurenda, 2002).

\subsubsection{Granularity of DWM}

Granularity (granular composition) characterizes the quantitative representation of individual particles, or group of particles of specific size, of the bulk material. Bulk material is a system (a set of particles with varying size) that can be sorted into classes depending on the size of its fractions. Table 2.2 displays classes according to STN 260070 and their corresponding fraction size.

Table 2.2: Classes of bulk material granularity according to STN 260070.

\begin{tabular}{lll}
\hline Class & Granularity $(\mathrm{mm})$ & Definition \\
\hline Very fine & $0.07-0.50$ & $\mathrm{~A}$ \\
Fine & $0.50-3.50$ & $\mathrm{~B}$ \\
Fine grain & $3.50-13.0$ & $\mathrm{C}$ \\
Medium grain & $13.0-75.0$ & $\mathrm{D}_{1}$ \\
Coarse grain & over 75.0 & $\mathrm{D}_{2}$ \\
Irregular & fibrous, diverse, irregular size & $\mathrm{E}$ \\
\hline
\end{tabular}

In Hejma\& et al.(1981), a different granular classification is given. According to this source, loose bulk wood materials are categorized as thick, medium thick, medium, fine, and very fine. Granularity of fractions can be seen in Table 2.3.

STN 260070 also states that dust is a fine fraction of bulk material including wood fractions from $0.1 \mu \mathrm{m}$ to $1000 \mu \mathrm{m}$. According to this standard, dust is classified by:

- very fine dust with particles from $0.1 \mu \mathrm{m}$ to $10 \mu \mathrm{m}$,

- fine dust with particles from $10 \mu \mathrm{m}$ to $100 \mu \mathrm{m}$,

- thick dust with particles from $100 \mu \mathrm{m}$ to $1000 \mu \mathrm{m}$. 
Table 2.3: Granularity of bulk wood materials (Hejma\& et al., 1981).

\begin{tabular}{ll}
\hline Granularity labeling & Fractions dimension $(\mathrm{mm})$ \\
\hline Coarse & $\mathrm{a}>1$ \\
Medium coarse & $0.5-1$ \\
Medium & $0.3-0.5$ \\
Fine & $0.03-0.3$ \\
Very fine & $0.001-0.03$ \\
\hline
\end{tabular}

The properties of wood dust with a particle size of $10 \mu \mathrm{m}$ to $100 \mu \mathrm{m}$ differs from other dust categories in that it has very slow sedimentation, remains airborne for a long time, is explosive and conductive, and tends to form clusters in humid conditions.

The most common method for defining the granular composition of bulk materials is to run them through screen "sieving," or a system of successive screens graduating with finer mesh sizes. There exist analytical methods for determining granular composition, including, for example, blowing or microscopic analysis. However, these methods tend to be slower and more expensive; therefore, the sieving method according to standards in Holm \& et al. (2008) is the favoured method.
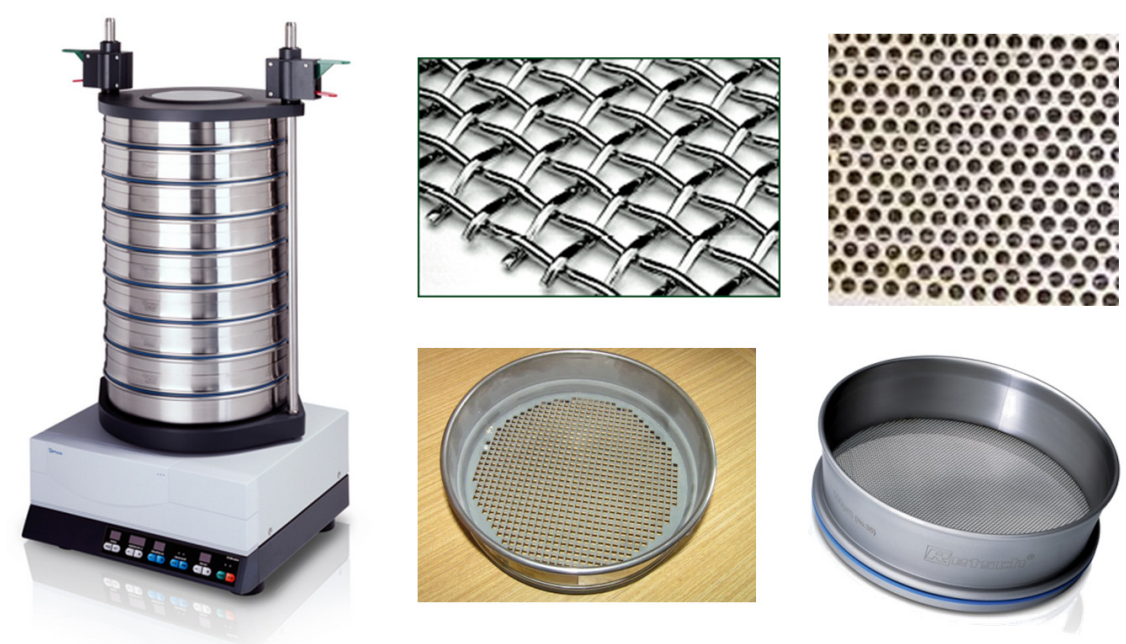

Figure 2.11: Sieving devices, different mesh types, and configurations (square or circular mesh) (Fischer; Retsch). 


\subsubsection{Moisture Content of DWM}

Moisture of wood material is determined through the weight of water $m_{v}$ within its volume. It is usually expressed in the form of absolute humidity or relative humidity of the wood material (STN EN 844-4).

When we discuss absolute humidity, we are defining the weight of water as a ratio to the weight of absolutely dry wood. When we define the weight of water as a ratio to the weight of wet wood, we are referring to the relative humidity. Absolute and relative wood humidity is expressed as a percentage $\%$ and is calculated through the following relations (STN 490103):

$$
\begin{gathered}
w_{a}=\frac{m_{v}}{m_{0}} .100=\frac{m_{w}-m_{0}}{m_{0}} .100(\%) \\
w_{r}=\frac{m_{v}}{m_{w}} .100=\frac{m_{w}-m_{0}}{m_{w}} .100(\%) \\
m_{w}=m_{0}+m_{v}(\mathrm{~kg})
\end{gathered}
$$

where:

$w_{a}$ - absolute humidity

$w_{r}$ - relative humidity

$m_{0}$ - weight of absolutely dry wood (weight of wood after drying at $103 \pm 2^{\circ} \mathrm{C}$ )

$m_{w}$ - weight of wood at a humidity of " $w$ "

$m_{v}$ - weight of water in the wood

The conversion of relative wood humidity to absolute, and vice versa, is possible using these equations (STN 490103):

$$
\begin{aligned}
& w_{a}=\frac{100 \cdot w_{r}}{100-w_{r}}(\%) \\
& w_{r}=\frac{100 \cdot w_{a}}{100+w_{a}}(\%)
\end{aligned}
$$

Relationship between absolute and relative humidity in wood is illustrated in Fig. 2.12. 
relative humidity $(\%)$

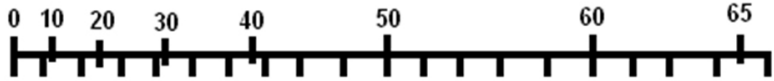

$\begin{array}{lllllllll}0 & 20 & 30 & 40 & 50 & 60 & 70 & 80 & 90100110120130140150160170180190\end{array}$

absolute humidity (\%)

Figure 2.12: Nomogram for the mutual conversion between absolute and relative humidity in wood (Požgaj \& et al., 1997).

The mechanisms holding moisture within wood are explained in STN 490103 and Požgaj \& et al.(1997), which discuss the chemical-bonded water, free water, and bound water.

Chemically-bonded water is a result of chemical compounds. They cannot be eliminated by drying, only by combustion. For this reason, it is included in the absolute dry state of the wood. Its content is determined through chemical analysis of wood, and its overall content represents about $1-2 \%$ of the dry wood matter. It has no effect on the physical/ mechanical characteristics.

Free water, known also as capillary water, mostly fills the lumen of the wood or sometimes the free space between cells in which it is drawn by capillary behaviour. It is only present in the wood when the cell walls are filled with bonded water. Its quantity ranges from the point of fibre saturation (PFS) to completely saturated wood with water. Its maximum quantity can exceed $100 \% w_{a}$. Its content depends mainly on the volume of lumen cells and, to a smaller degree, the intercellular spaces in the wood matter. Although it has some effect on the physical/mechanical characteristics of the wood, it is not nearly as influential as bound water.

Bound water, known also as hygroscopic water, is located in the cell walls and is bonded by hydrogen in the $\mathrm{OH}$ hydroxide group of the amorphic part of the cell and hemicellulous. The water bound within wood has moisture content from $0 \%$ to PFS, whose average humidity value for the wood used in this work was 30\% $w_{r}$ (Požgaj\& et al., 1997). PFS is defined as a wood state consisting of bound water with the cell walls saturated such that the free spaces in the cell are filled with water. The moisture content in the wood used in this paper was 22 to $35 \%$ wr at the PFS (Požgaj \& et al., 1997). It depends mainly on the species of wood, methods of extraction, and other effects. Bound water has the greatest, and therefore most important, effect on the physical/mechanical characteristics of the wood matter. The boundary between bound water and free moisture is determined through the limit of cell wall saturation (LCWS) or hygroscopic limit (HL). Such a state is defined when the cell walls are fully saturated while the lumen does not consist of any free water. The difference between LCWS and HL is defined by the environment that the wood is in. LCWS refers to water in a liquid state, whereas the water in the $\mathrm{HL}$ is in a gaseous state. At $15-20^{\circ} \mathrm{C}$, 
both variables have approximately the same moisture content (about $30 \% w_{r}$ ), but unlike LCWS, HL is dependent on the surrounding temperature and with growing temperature decreases (Požgaj \& et al., 1997).

In practice, the degree of moisture in the wood is distinguished by (Požgaj \& et al., 1997):

1. Wet, long-term exposure to water (over $100 \% w_{r}$ );

2. Freshly-cut (50 to $100 \% w_{r}$ ), in some cases up to $180 \% w_{r}$ depending on wood species

3. Air dry (15 to $\left.20 \% w_{r}\right)$

4. Room dry (8 to $10 \% w_{r}$ )

5. Absolutely dry, dried at $103 \pm 2{ }^{\circ} \mathrm{C}$

Wood saturation is dependent on the moisture level of the wood. It is the ability for the wood, thanks to it porous structure, to draw in liquid water. The amount of free water is dependent mainly on the volume of the pores within the wood. The saturation rate of the core is slower than for the bark (Fig. 1.1). With increasing density, saturation rate of wood decreases. Higher temperatures accelerate the saturation rate of the wood. Moisture, as a contributing factor to the densification process, will be given further attention when we discuss parameters affecting the quality of briquette.

\subsubsection{Bulk Density of DWM}

In discussing the particulate matter of wood, it is necessary to recognize one property of its bulk, which is its angle of repose. The angle of repose is a characteristic element that defines the properties of the particulate matter. Its range of values can be, theoretically, from zero to ninety degrees. The closer its value is to zero, the less cohesive the particulate matter is and vice versa.

However, in our case (pressing of DWM) the bulk density is more important. The bulk density is defined as the ratio of particle mass $m$ to its volume $V_{S}$ (Dzurenda, 2002).

$$
\rho_{S}=\frac{m}{V_{S}}\left(\mathrm{~kg} \cdot \mathrm{m}^{-3}\right)
$$

For the measurement of bulk density, as well as the angle of repose for that matter, we can use Gary's apparatus (Fig. 2.13). The test is very simple. A 1000ml chute with open bottom fills a sample container $350 \mathrm{~mm}$ below the chute by means of free falling particles and gravity. After the sample is completely filled, the top is levelled and the specimen is then weighed. With known volume and weight of the specimen, we can use Equation 2.8 to determine the bulk density of the wood matter. 


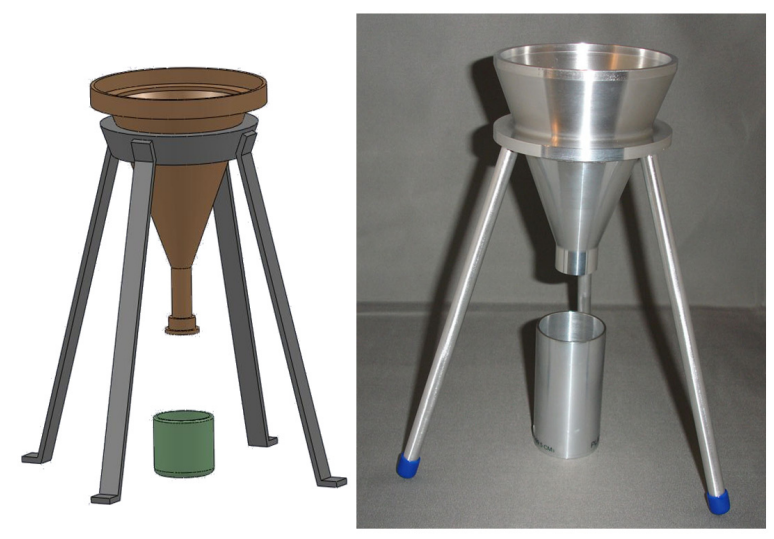

Figure 2.13: Gary’s apparatus for measuring bulk density of particulate matter (Dzurenda, 2002).

Testing for the angle of repose utilizes the same apparatus, except the sample container is removed and the particles are allowed to drop freely onto the flat surface below, creating a cone-like shape. After pouring, the height and diameter of the poured bulk are taken. Cohesive substances have a higher angle of repose, while noncohesive substances have a lower angle. The lower the angle, the easier the material will pour allowing for reliable dosages of material. However, a low angle of repose represents materials that are less cohesive, requiring more area needed for their storage, which can be problematic.

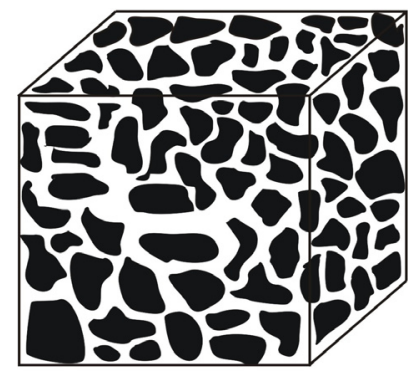

Bulk density

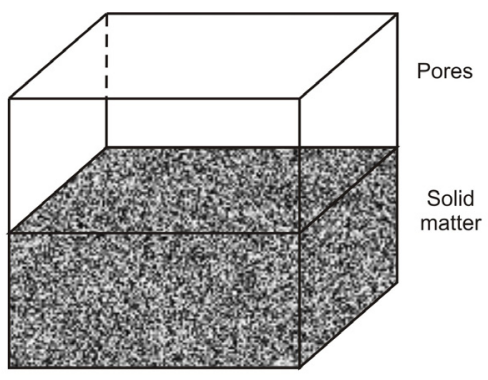

Volume weight

Figure 2.14: Illustrative representation between bulk density and volume weight (Simanov, 1995).

Bulk density is dependent on the properties of the bulk material (wood species, granularity, moisture content). The approximate dependence between wood density and bulk density of DWM can be expressed by: 
$\rho_{\mathrm{S}}=(0,3 \div 0,5) \cdot \rho\left(\mathrm{kg} \cdot \mathrm{m}^{-3}\right)$

Table 2.4: Bulk density of some wood materials with a moisture content of $w_{r}=10 \%$ (Dzurenda, 2002).

\begin{tabular}{ll}
\hline DWM & Bulk Density $\left(\mathrm{kg} \cdot \mathrm{m}^{-3}\right)$ \\
\hline Spruce shavings & $80-124$ \\
Spruce sawdust & $113-166$ \\
Beech sawdust & $195-218$ \\
Wood chips & $238-259$ \\
Dust from sanding & $256-279$ \\
\hline
\end{tabular}

\subsubsection{Spacing and Porosity of DWM}

Gaps between wood particulate matter in contact with each other in bulk can be represented as pores. Since solid particles consist of surface pores and internal pores, porosity of the bulk material represents the volume of these pores or volume around the particles.

These parameters are not only important for particulate matter (represented by a volume that can be filled with a liquid phase) but also for storage, transportation, burning processes, and automated incineration. Spatial porosity impacts effective storage, packaging, transport, and manipulation of particles with varying sizes.

Spacing effects (Bejda \& Miklušová, 2002):

- Shape of the particle - for example, a cubic configuration has a spacing of $\varepsilon=0$, whereas a spherical particle will never obtain zero spacing.

- Fraction composition - compacting biofuels is monodisperse (same size and identical shape. Spacing larger than polydisperse substances, which fill their spaces with dust.

- $\quad$ Particle organization - spherical particles of identical diameter have six configurations and six different values of $\varepsilon$. The spacing depends also on the number of contacts between particles. At the walls, spacing is greatest.

Calculating spacing can be done using Equation 2.10, where $V_{\varepsilon}\left[\mathrm{mm}^{3}\right]$ is the volume of the spaces, and $V_{t}\left[\mathrm{~mm}^{3}\right]$ is the overall volume:

$$
\varepsilon=\frac{V_{\mathrm{e}}}{V_{\mathrm{e}}+V_{t}}
$$


Spacing is almost impossible to determine for bulk materials in, for example, a sac or container. It is very difficult and work-intensive to determine the orientation of each individual briquette in space. Problems are thus necessary to simplify and place objects into bricks whose dimensions represent the maximum dimensions in space. Significant inconsistencies in particle shapes can also greatly affect filling of the spaces.

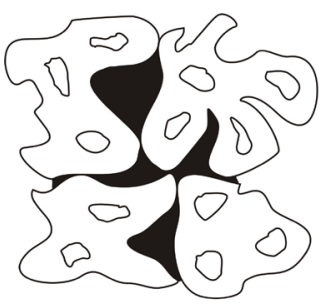

spacing

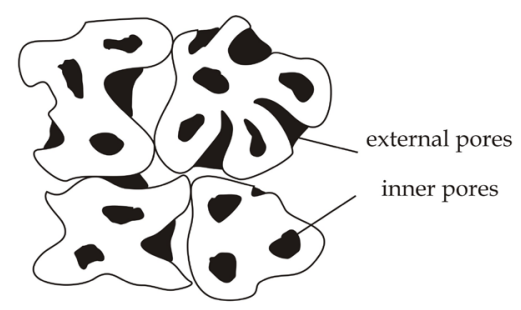

porosity

Figure 2.15: Illustrative representation of spacing and porosity (Pietsch, 1991).

\subsubsection{Compressibility of DWM}

The compressibility of DWM is defined as the ability for the bulk material to change its volume with respect to an externally applied force. It is characterized by the coefficient of compressibility $\beta_{S}$, which gives the relative change in volume for the unit change in applied pressure.

$$
\beta_{S}=\frac{1}{V_{o}} * \frac{d V}{d p}\left(\mathrm{~m}^{2} \cdot \mathrm{N}^{-1}\right)
$$

Compressibility is one property that, in pressing, affects the process. Compressing DWM densities decreases the size and porosity of the bulk material while the volumetric weight increases through the application of external force. The simplest way to evaluate the compressibility of a particulate matter is by uniaxial compression (pressing). A specimen is prepared with a so-called oedometer. The sample is pressed by a force and the corresponding compressibility curve, which describes the dependence of compression for of the pressed sample, is obtained. 


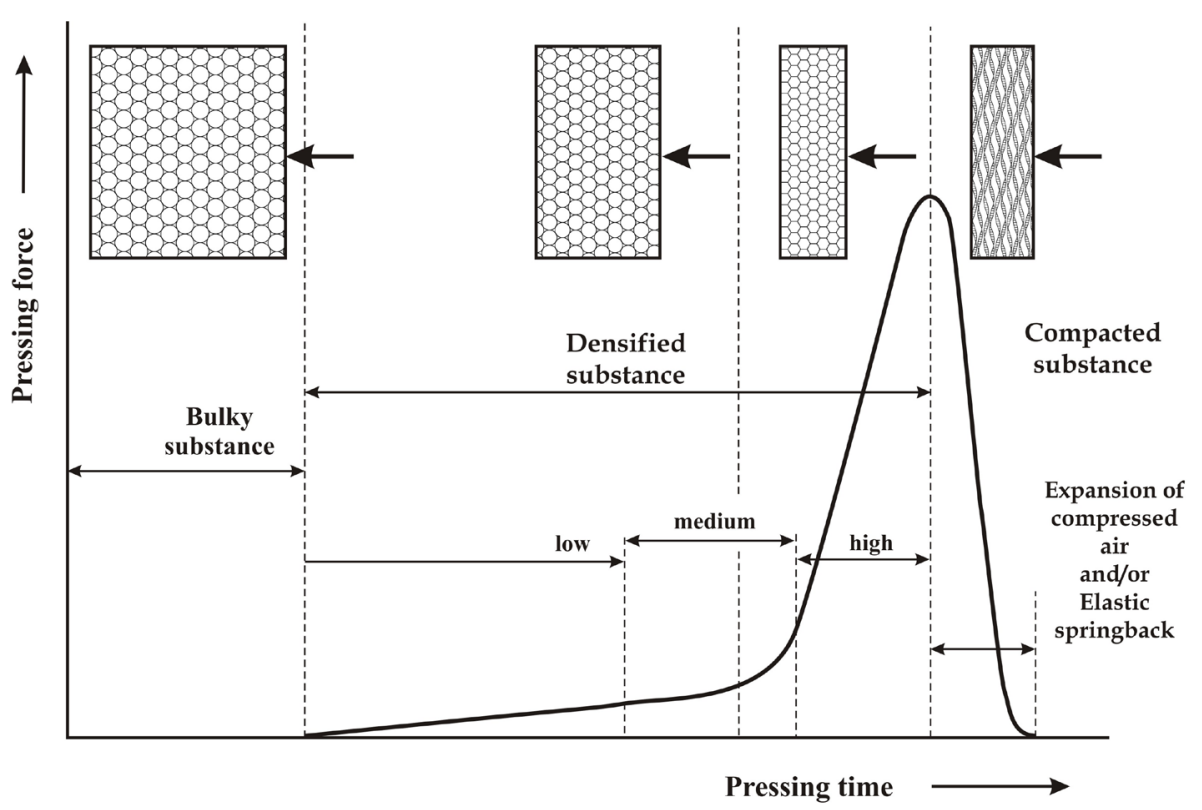

Figure 2.16: Change in wood mass depending on force applied (Pietsch, 2002).

Under the applied pressing pressure, solid particles of the material are pushed closer to each other until they create a strong adhesive bond. In the upper part of the previous figure, four sketches representing the change in structure of a bulk material during compaction are shown. The accompanying effects of applying high pressure to the particles are a change in the shape and size of the particles and therefore a change in the overall volume. In the bottom part of the previous figure, the growth of pressing force with respect to time assumes that the movement of the piston is constant until the maximum value. After obtaining the maximum value, the movement of the piston is in the opposite direction and also constant. In the first step of densification, the particles within the material regroup and, due to lower forces, do not change shape and size of the particles. In the second step, where the pressing force begins to rise, fragile particles break and elastic particles deform (sketch 3 and 4). It is necessary to consider two interesting properties which affect and limit the densification process as well as the performance of the pressing machine. Pressing excess air within the pores and elastic deformation affect the densification and compressibility of the particles. They cause specific cracks and weaknesses in the briquette which can lead to breakage of the briquette. The gradual increase in pressing pressure changes the size of the particles until deformation, reducing porousness. The degree to which the particles deform is given by the compressibility of individual types of bulk wood materials. Compressibility is one of the properties which, when pressing, affects the whole process. The maximum pressing force is defined by the structural and 
kinematic properties of the pressing machine, and therefore it is necessary to design a densification process for wood with respect to the machine's capabilities.

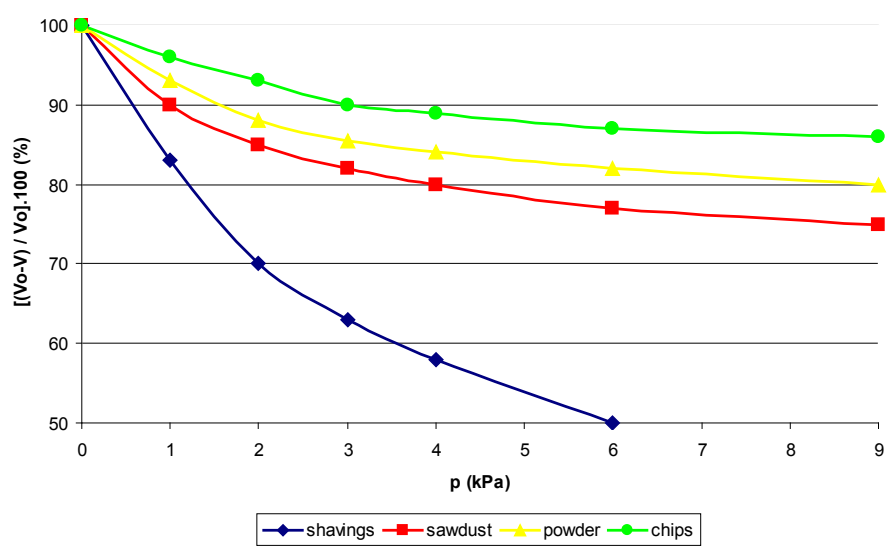

Figure 2.17: Compressibility of some types of wood materials (shavings, dry saw dust, wood powder, chips) (Dzurenda, 2002).

On the previous figure, we can see the differences in compressibility for various types of bulk wood materials. The compressibility coefficient of wood matter consisting of isometric particles have values of $\beta_{\mathrm{s}}=0.75-0.85$ and $\beta_{\mathrm{s}}=0.50$ for wood shavings. The compressibility of chips, wood dust, dry sawdust, and shavings with respect to pressure can be seen in Fig. 2.17.

\subsection{Evaluating the Quality of the Briquettes with Respect to Standards}

As for any area of research, densification of wood biomass into a biofuel requires us to define the quality of production. Solid biofuels are characterised by a wide range of quality, defining their physical and chemical composition, such as the size of the particles, moisture content, heating potential, or the sulfur, chlorine, nitrogen, and potassium content. It is therefore very important to define the properties and standardize parameters of solid biofuels (ECS, 2013a; 2013b). Standardization of solid biofuels is a very important and necessary prerequisite to expand the market for solid biofuels. Stating some standards for solid biofuels can contribute to simplifying the offering of biofuel, its conversion into energy, establish biofuel in the market, and reach an economical understanding for price of fuel. Furthermore, standardization can guarantee that each fuel fulfills the demands for combustion and therefor guarantees 
the environmental cycle minimizes emissions of dangerous substances into the atmosphere and environment. It also defines the connection between production and operational machines for energy conversion (furnaces, etc.). Standardization helps reduce potential expenses in the system and thus minimizes the overall investment into such systems.

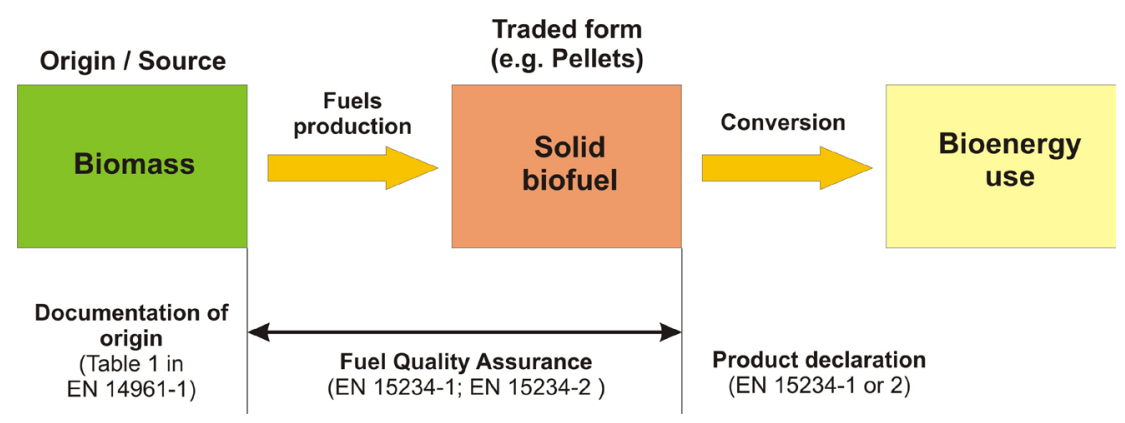

Figure 2.18: Effects of Technical Standards in the production of solid biofuels (STN EN 14588).

Currently, many Standards exist for solid fuels. Legislation, manuals, and technical standards define the requirements for the production, energy uses, and markets for solid biofuels. They are necessary for sellers as they objectively clarify the rules and simplify the quality requirements in the market. They also help create markets by making all relevant information readily available. Control and interpretation is based on quality requirements, which also increase the competition of this fuel and categorizes the quality of the fuel, storage, and supply (Šooš \& et al., 2010).

It is important to comment that the European Union Standards are defined for solid biofuels under CEN 14588 and CEN 14961, for example. These standards define biofuels, raw materials for production, indicators of quality, procedures for determining properties of the biofuel, and the rating system for the final product quality. In more developed countries (countries with a higher production of biomass), other standards existed even before those put in place by the EU. These national standards were the basis for creating the newly-formed EU Standards. In Slovakia, the market for biofuels is gradually increasing. It is therefore necessary to be up to date on the current European Union Standards. Each valid EU Standard must be implemented into Slovakian Standards. Presently, biofuel production in Slovakia, as well as abroad, adhere to current legislation; however, some initial technical standards of some EU states are still used. Therefore, current as well as past EU Standards will be discussed. 


\subsubsection{Dividing of Briquettes According to Standards of Some EU Countries}

Before the development and the acceptance of current EU legislation (up to 2005), most standards were defined at a national level for countries such as Austria, Germany, and Sweden. Adapting these standards was easy for other EU countries. A briquette/ pellet as a final product from the densification process, further referred to as a biofuel, must satisfy some specific conditions stated in the Technical Standards. In Europe, Germany, Austria, Sweden, and Italy, Standards exist for the production of special solid biofuels. More Standards exist to supplement these basic Standards.

In Austria, a national Standard exists defining the quality of the production, storage, and transport of wood pellets and briquettes, more specifically known in Ö-Norm M 7135:

- ÖNORM M 7135 - briquettes from natural wood, pellets, and briquettes; requirements and legislation for testing;

- ÖNORM M 7136 - briquettes from natural wood and wood pellets; ensuring quality in the transport and logistics to the end user;

- ÖNORM M 7137 - Briquettes from natural wood, wood pellets; conditions for the storage of pellets at the end users facilities;

Certificates for solid high-grade biofuels in Germany were established on the DIN 51731 and DIN Plus Standards (DIN 51731; DIN Plus). The certification is based on initial evaluation and conformity, such as routine monitoring. Monitoring is restricted to the yearly repetition of tested products in a laboratory certified by DIN CERTCO (German Institute for Standards).

- $\quad$ DIN 51731 - Testing of solid fuels; briquettes from natural wood; requirements and testing;

- $\quad$ DIN Plus - certificate scheme; wood pellets for use in small furnaces;

- DIN 51731 defined the term "briquette" as a fuel artificially created by pressing bulk materials without any added substances and suitable for burning.

The basic dimensions of a briquette or pellet according to DIN 51731 as well as Ö-Norm M 7135, are given in Tab. 2.5. The limit density of pellets and briquettes is $1-1.4$ $\mathrm{kg} . \mathrm{dm}^{-3}$, with a moisture content below $12 \%$, ash content below $1.5 \%$, and heating energy from 17.5 to $19.5 \mathrm{MJ}^{\mathrm{kg}}{ }^{-1}$. Further addressed in the Standards are the bulk density, abrasiveness, chemical limits, trace elements, and emissions. At the present, they are produced exclusively for energy (Fig. 2.19 and 2.20). Standards state that extrusions up to $25 \mathrm{~mm}$ in diameter are considered pellets, and anything larger is considered a briquette. Briquettes are formed into cylinders, blocks, and parallelograms. They are typically used in larger automatic furnace systems or home furnaces and fireplaces without any atomization. 
Table 2.5: Dimensions of wood pellets/briquettes (HP) and from cortex wood (RP) produced in line with DIN 51731 and Ő-Norm M 7135.

\begin{tabular}{|c|c|c|c|}
\hline Standard & Size group & Diameter $D_{N}$, height or thickness $(\mathrm{mm})$ & Length $(\mathrm{mm})$ \\
\hline \multirow{6}{*}{ 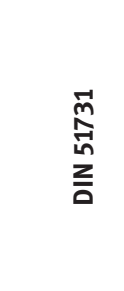 } & HP 1 & $>100$ & $>300$ \\
\hline & HP 2 & 60 to 100 & 150 to 300 \\
\hline & HP 3 & 30 to 70 & 100 to 160 \\
\hline & HP 4 & 10 to 40 & $<100$ \\
\hline & HP 5 & 4 to 10 & $<50$ \\
\hline & HP 1 (pellets) & $4 \leq D_{N}<10$ & $\leq 5 \times D_{N}$ \\
\hline \multirow{5}{*}{ 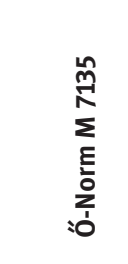 } & HP 2 & $10 \leq D_{N}<40$ & $\leq 4 \times D_{N}$ \\
\hline & HP 3 (briquettes) & $40 \leq D_{N}<120$ & $\leq 400$ \\
\hline & RP 1 (pellets) & $4 \leq D_{N}<10$ & $\leq 5 \times D_{N}$ \\
\hline & RP 2 & $10 \leq D_{N}<40$ & $\leq 4 \times D_{N}$ \\
\hline & RP 3 (briquettes) & $40 \leq D_{N}<120$ & $\leq 400$ \\
\hline
\end{tabular}

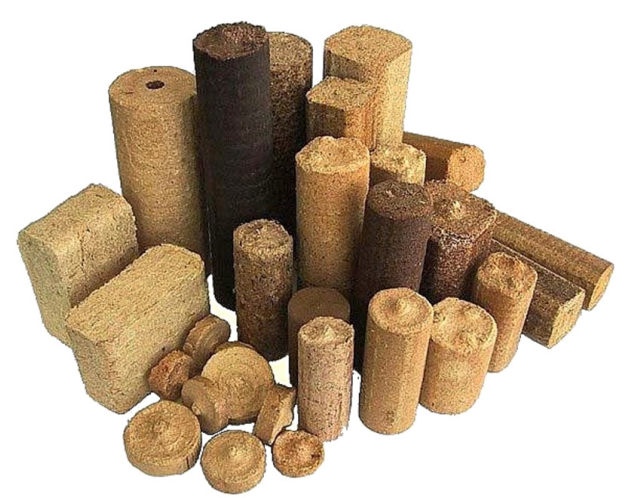

Figure 2.19: Examples of varying shapes of briquettes from wood.

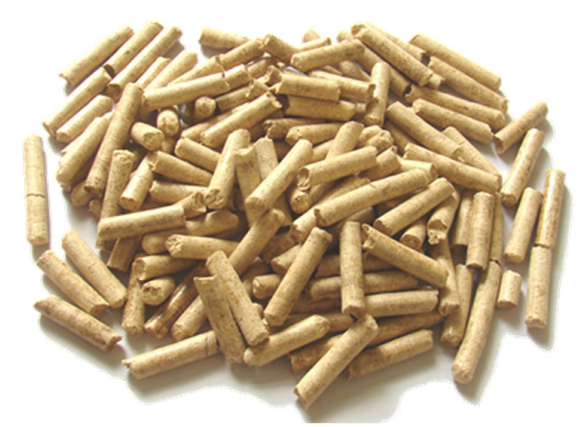

Figure 2.20: Examples of pellets from wood. 
The research in this work will focus on the production of briquettes up to $20 \mathrm{~mm}$ in diameter. The measurement stand is described in detail throughout this monograph. Briquettes can be created not only from wood but other types of materials such as phytomass (Fig. 2.21).

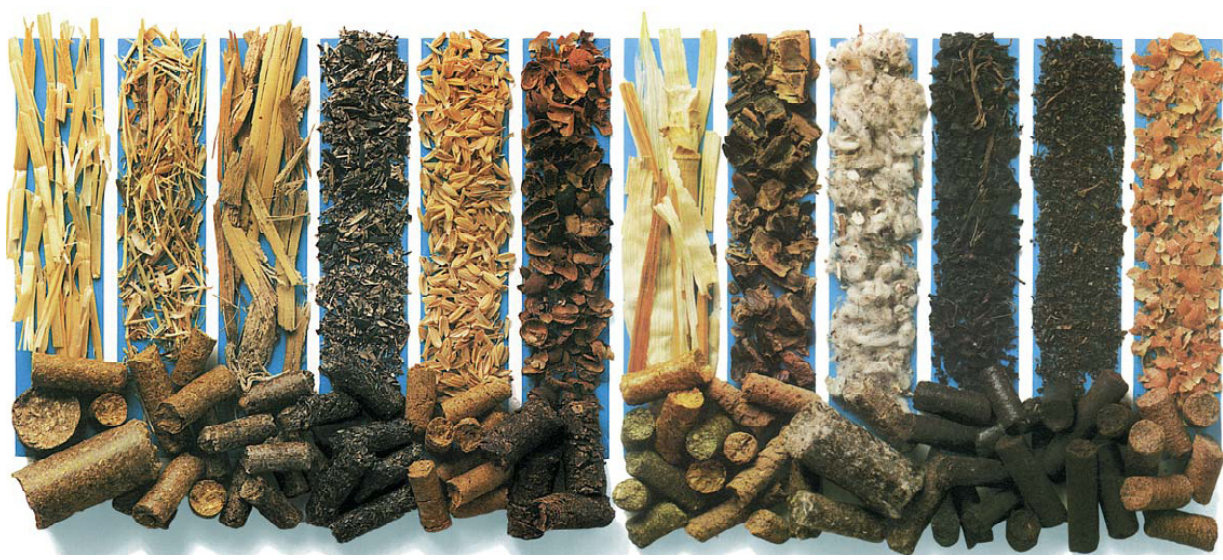

Figure 2.21: Briquettes from various types of materials (Melcer \& Melcerová, 1985).

In Sweden, a wide variety of Standards that define the qualitative parameters of briquettes and pellets, evaluate quality, and specify testing methods to determine various parameters have been adopted. Standards for briquettes describe three classes, which differ mainly in the size and ash content. Some basic technical standards from Sweden are:

- SS 187106 - Solid biofuels and peat - Terminology;

- SS 187120 - Solid biofuels and peat - Pellets - Classifications;

- SS 187123 - Solid biofuels and peat - Briquettes - Classification;

In Switzerland, the qualitative properties of solid biofuels were controlled according to Swiss National Standards SN 166000 (Testing of solid fuels - compressed untreated wood, requirements, and testing), which was identical to the German DIN 51731 standard.

In Great Britain, briquettes were introduced through a project organized by the Department of Trade and Industry (DTI). One of the results of this project was the categorization of possible uses of briquettes and furnaces utilizing them for homes (up to $15 \mathrm{~kW}$ ). This category was developed to the extent of measurement and evaluation of biofuel parameters at the national level. 
Similarly, Italian Standards include CTI-R04/5 (Caratterizzazione del pellet a finienergetici: quality parameters of briquettes for energy); CTI-R03/1 (Biocombustibili: specifiche e classificazione: biofuel - specification and classification); UNI/TS 11263 (Technical specification for the characterisation of briquettes for energy). France also had its own standards, including NF label (NF Granules Biocombustibles: French standard for granular biofuels). In Norway, there is the NS 3165 (Biofuel - Cylindrical pellets of pure wood - Classification and requirements. In Russia, there is the GOST 23246 - 78 (Crushed wood: Terms and definitions). Additionally, Finland has SFS 5875 (Solid recovered fuel: Quality control system).

Even though Denmark is a large user of pellets, there has never been any national Standard implemented for their use. Only the "Legislation for biomass" (BEK no.638 from 03.07.1997), in which it is only stated that briquettes must be made purely of wood with no additional substances, existed.

Other European countries (Estonia, Latvia, Greece, Cyprus, Poland, Portugal, Spain, Malta, Holland, Luxemburg, Belgium, Ireland, Hungary, Slovakia, Czech Republic, Slovenia, and Bulgaria) have never implemented any national level Standards for biofuels mainly because:

- the biomass market (mainly in briquettes) in these countries was very young;

- these countries waited for legislative implementations from the EU.

\subsubsection{European Technical Standards for Briquettes Quality Evaluating}

STN CEN 15357 applicable also in Slovakia, defines the following basic terms:

- "biofuel" - a fuel made, directly or indirectly, from biomass.

- "briquette" - a block or cylinder from solid alternative fuel made by the agglomeration of bulk material.

- " pellet" - a piece of solid alternative fuel made from agglomerated bulk material in a pressing form (disk or drum) with a diameter equivalent to $<25 \mathrm{~mm}$.

The now valid standard in Slovakia defined by STN CEN 14588 defines these basic terms:

- "solid biofuel" - a solid fuel made, directly or indirectly, from biomass.

- "biofuel briquette" - compressed biofuel made with or without additives in the pressing process in the form of cubes or cylinders, obtained through pressing biomass powder. Raw materials for briquettes can be wood biomass, herbal biomass, fruit biomass, and other types of biomass.

- "biomass pellets" - compressed biofuel made from powder biomass with or without pressing additives most commonly in cylindrical form of random length typically from 5 to $30 \mathrm{~mm}$ with broken ends. 
Technical standardisation of solid biofuels is administered by the European committee for standardisation CEN/TC 335 (ECS, 2013a; 2013b).This committee was formed in 2001 and since then has gradually prepared new standards for the area of solid biofuels. The European committee for CEN standards has prepared 38 published Standards for solid biofuels (CEN-TC 335). These Technical Standards are proceeded by all existing national Standards of European countries and member states of the EU, which must be implemented into each state's national Standards.

In Figure 2.22 and 2.23, valid EN Standards for solid biofuels regarding the size of briquettes and pellets are shown. In general, an extrusion with a diameter of $D<\varnothing 25$ $\mathrm{mm}$ is known as a pellet while briquettes are defines as anything larger. Further size and shapes of the briquette are defined within the six-part set of Standards STN EN 14961: Solid biofuel, specifications, and class.

\begin{tabular}{|c|c|c|}
\hline & $\begin{array}{l}\text { Origin: } \\
\text { According to } 6.1 \text { and Table } 1\end{array}$ & $\begin{array}{c}\text { Woody biomass (1); } \\
\text { Herbaceous biomass (2); } \\
\text { Fruit biomass (3); } \\
\text { Blends and mixtures (4). }\end{array}$ \\
\hline & Traded Form (see Table 2): & \multirow{3}{*}{$\begin{array}{c}\text { Pellets } \\
\text { Diameter (D) and Length }(L)\end{array}$} \\
\hline & Dimensions (mm) & \\
\hline D 06 & $6 \mathrm{~mm} \pm 1.0 \mathrm{~mm}$, and $3.15 \leq \mathrm{L} \leq 40 \mathrm{~mm}$ & \\
\hline D 08 & $8 \mathrm{~mm} \pm 1.0 \mathrm{~mm}$, and $3.15 \leq \mathrm{L} \leq 40 \mathrm{~mm}$ & \multirow[b]{4}{*}{ Figure 3: Dimensions (mm). } \\
\hline D 10 & $10 \mathrm{~mm} \pm 1.0 \mathrm{~mm}$, and $3.15 \leq \mathrm{L} \leq 40 \mathrm{~mm}$ & \\
\hline D 12 & $12 \mathrm{~mm} \pm 1.0 \mathrm{~mm}$, and $3.15 \leq \mathrm{L} \leq 50 \mathrm{~mm}$ & \\
\hline D 25 & $25 \mathrm{~mm} \pm 1.0 \mathrm{~mm}$, and $10 \leq \mathrm{L} \leq 50 \mathrm{~mm}$ & \\
\hline
\end{tabular}

Figure 2.22: Specifications for pellet properties - excerpt from the STN EN 14961-2 Standard.

These Slovak Standards are based on existing European Technical Standards. At present, most European Standards for solid biofuels have been adapted. In the next table, a comparison of briquette parameters, which are published in some national and EU Standards, is shown.

\subsubsection{Indicators of Quality and Evaluation of Briquettes with Respect to Standards}

Solid, high-grade biofuels are considered high quality if they fulfill the requirements defined within Technical Standards. Technical Standards define multiple parameters (indicators), which are used to evaluate and monitor the quality solid, high-grade 


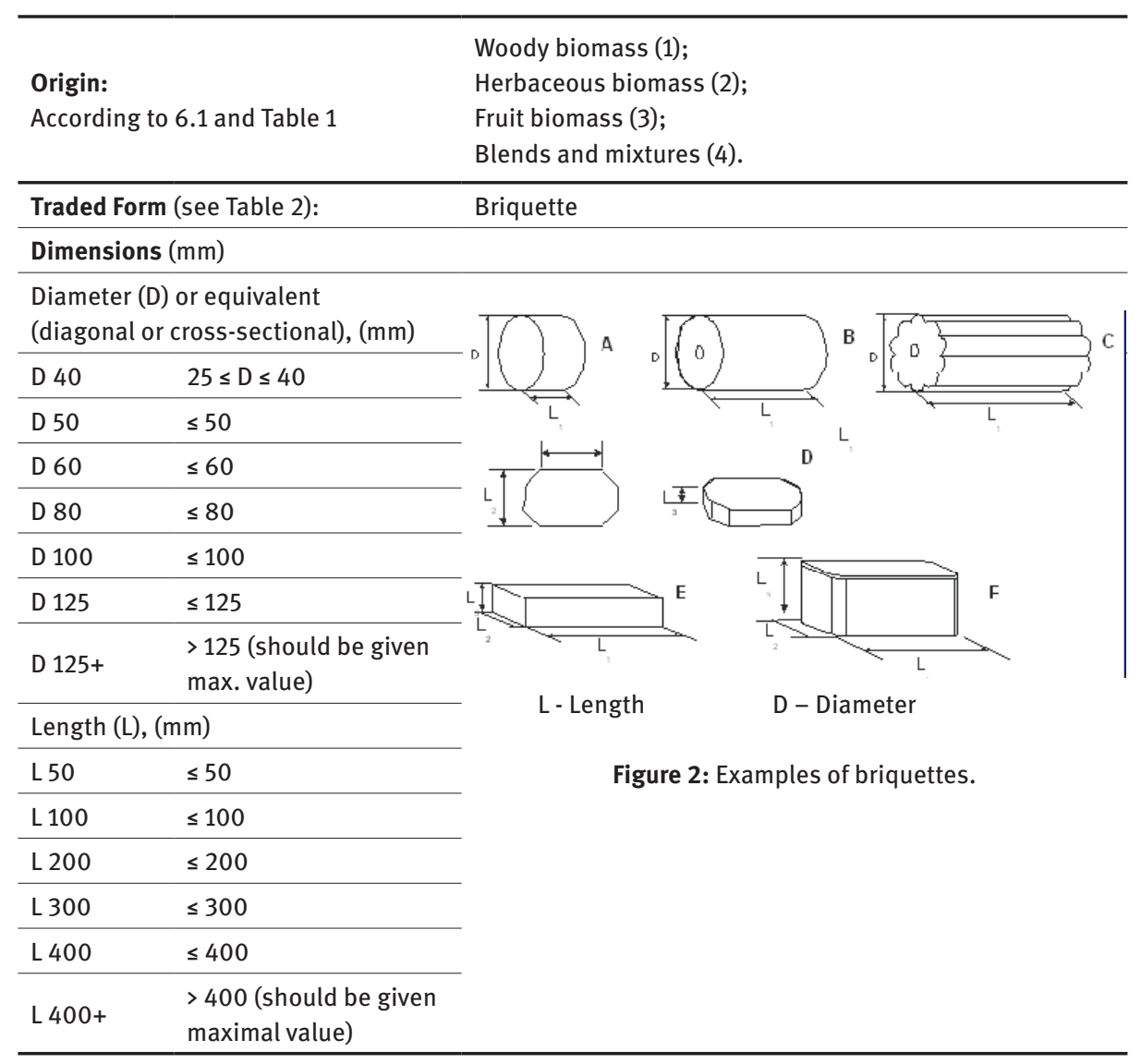

Figure 2.23: Specifications for briquette properties - excerpt from the STN EN 14961-3 Standard.

biofuel (SHGB). Each monitored parameter/indicator of quality is precisely described, with a procedure for its identification, and for each parameter the precise limit for its value is defined. If a briquette falls within these limits, it can be considered a quality product. However, this limit for quality is valid only in cases where the solid biofuel satisfies all other monitored quality indicators (mechanical and chemical/thermal).

Quality indicators of solid biofuels can be divided into two basic groups (Lehtikangas, 2001; Rönnbäck, Sager \& Alakangas, 2012):

- physical-mechanical indicators

- chemical-thermal indicators. 


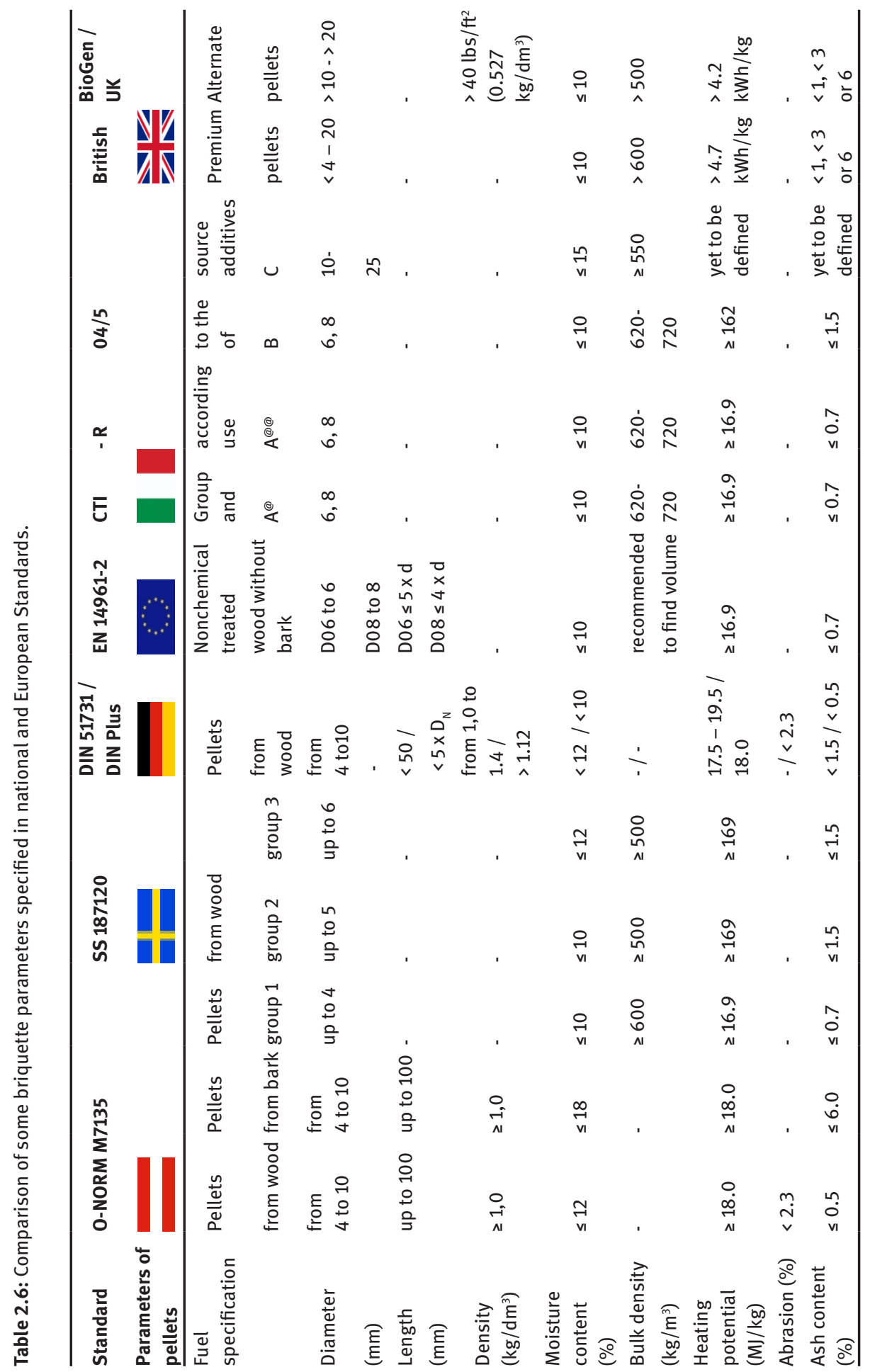




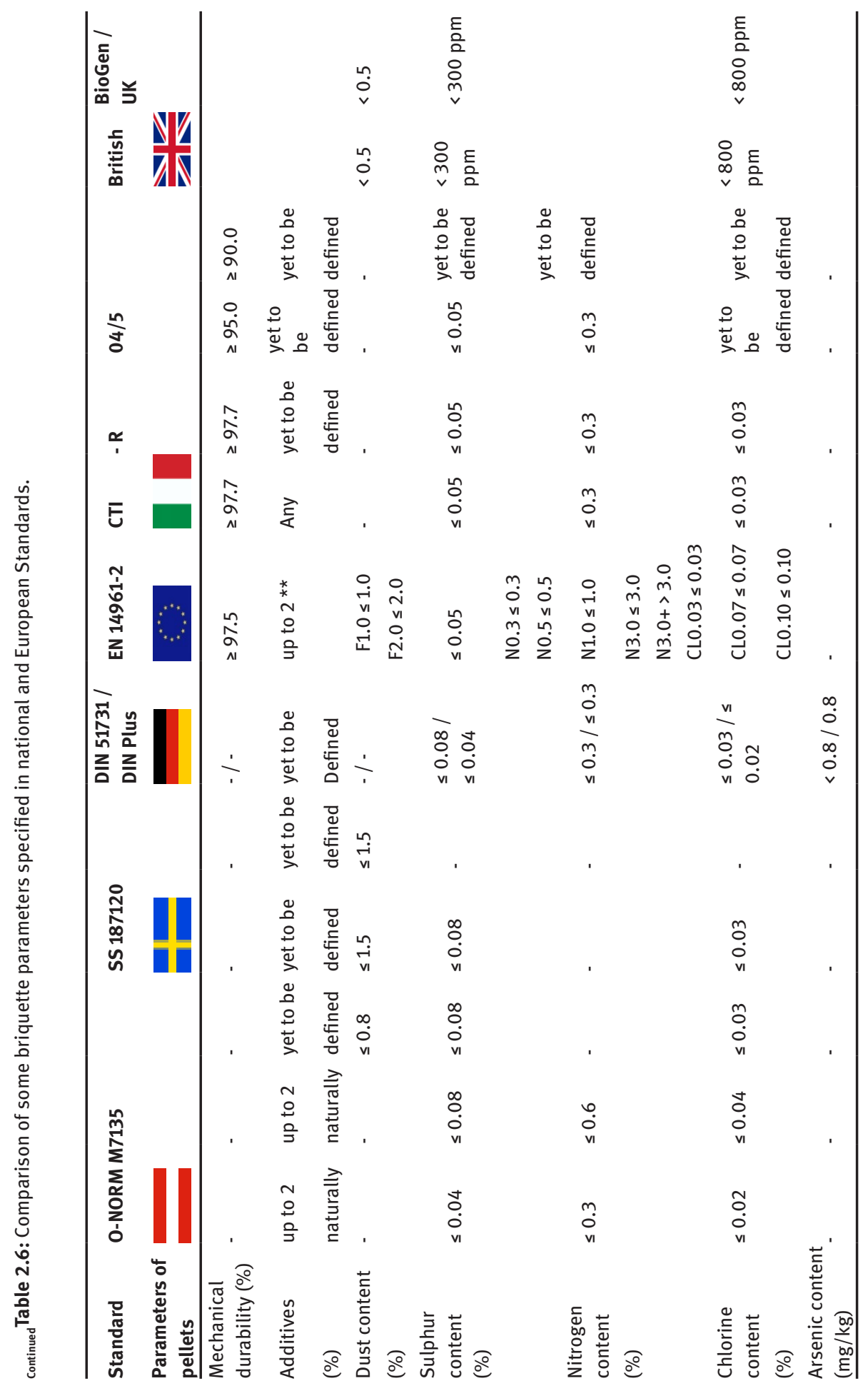




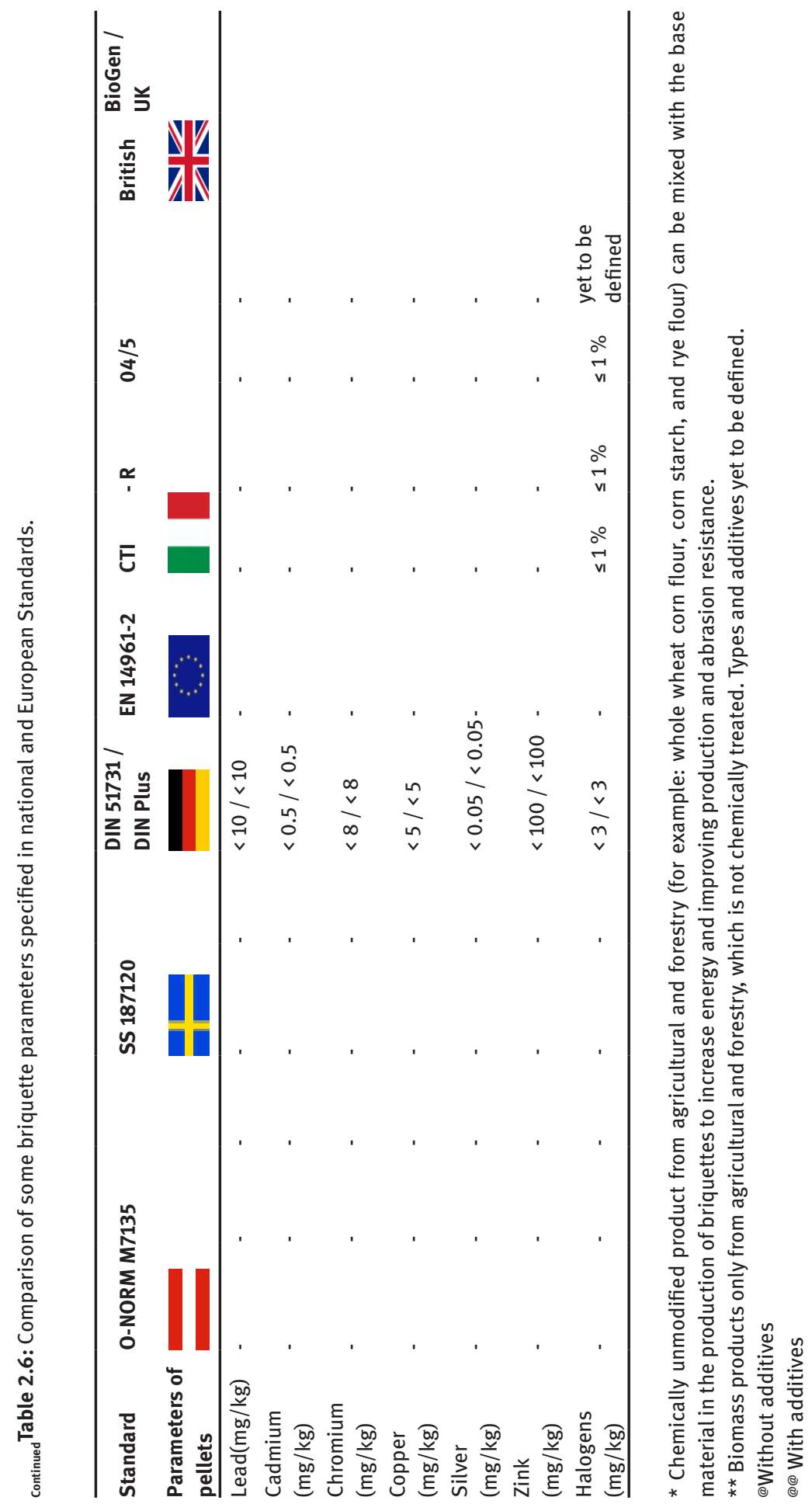




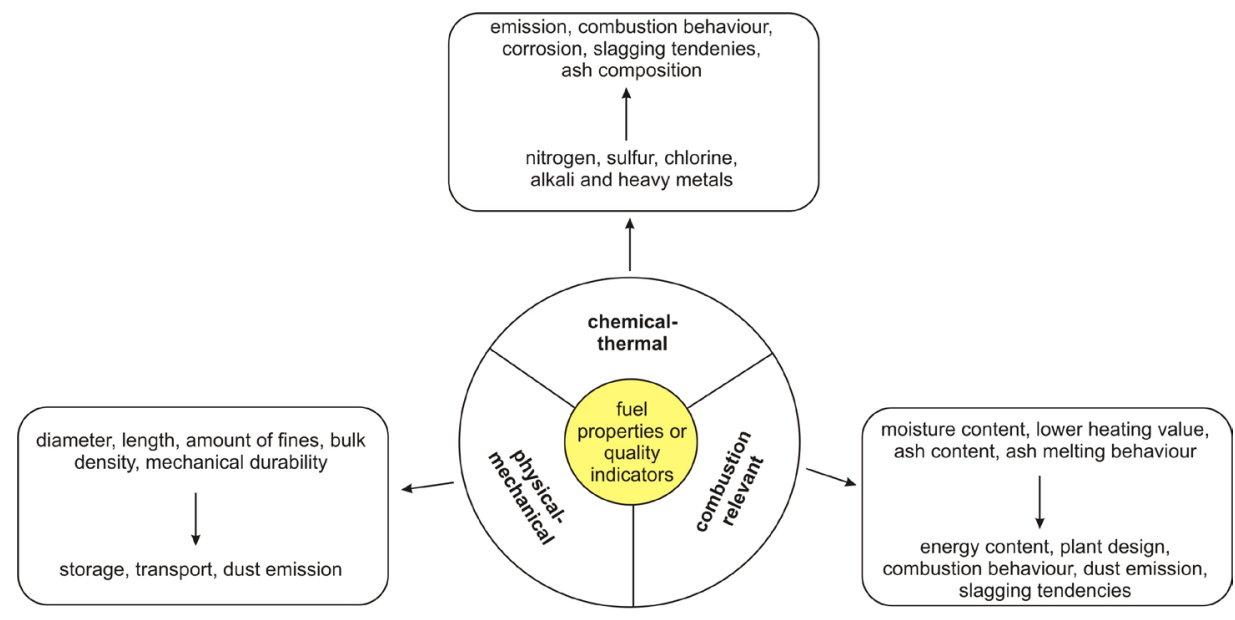

Figure 2.24: Property effects of fuel in the combustion process (EN Plus Handbook, 2013).

The growth phase and origin of the biomass affect mainly the chemical-thermal properties, whereas the material processing phase most importantly affects the mechanical properties of the final product.

Before the implementation of European Standards (EN 14961) for the evaluation of SHGB, every producer and seller within the EU focused on achieving the previously mentioned Austrian (Ö-NORM M 7135) and German (DIN 51731 a DIN Plus) Standards. These Standards defined, in more detail, requirements for production and indicators of briquette quality.

ÖNORM M 7135:2000 Compressed wood or compressed bark in natural state-pellets and briquettes-- requirements and test specifications. Vienna, Austria: ÖsterreichischesNormungsinstitut

Description: compressed wood in its natural state - pellets and briquettes requirements and specification of tests. The implementation of this Standard prompted the wood-working industry. It is a unified standard for pellets and briquettes. This Standard restricts use and defines basic terms and requirements for extrusions and described testing methods.

DIN 51731:1996 Testing of solid fuels - compressed untreated wood, requirements, and testing. Berlin, Germany: DeutschesInstitutfürNormung

Description: solid fuel testing - pressing wood, requirements, and testing. This Standard contains the definition for wood extrusions, their labelling, dimensions, allowable emissions, and testing procedures. This Standard divides briquettes from wood waste into five size groups. 
DIN Plus:2002 Certification Scheme - Wood pellets for use in small furnaces. Berlin, Germany: DIN CERTCO-Gesellschaft fur KonformitatsbewertungmbH

Description: certification scheme - wood briquettes/pellets for use in small heating systems. This Standard presents a new certification scheme and combines the requirements according to the German DIN 51731 and Austrian ÖNORM M 7135 Standards. The requirements in relation to maximum property values of some products change compared to the certification of wood briquettes/pellets according to the DIN testing scheme.

The sixth chapter in ÖNORM M 7135, seventh chapter in DIN 51731 and part 3.11 of DIN Plus are dedicated to pellets. Therefore, we will further discuss these chapters. The detailed description for quality assurance in the areas of logistics, transport, and storage is written in ÖNORM M 7136. The storage requirements at the user facility is described in ÖNORM M 7137.

As discussed above, we can divide the quality indicators of the briquettes into two categories. The first concerns the chemical-thermal indicators of quality. Select Technical Standards of some European countries include the ash content, heating value, moisture content, and chemical and trace elements. The second category consists of so-called mechanical indicators of quality including parameters such as briquette density and abrasion resistance. The first category is essentially dependent on the type of compressed material (its properties and chemical composition). The second category depends on several parameters, which also include the experimental research described in this work. These parameters are mentioned in further chapters. Mechanical indicators are very important and decisive to this research, and therefore their descriptions will be discussed.

Currently, the most important Standard for solid biofuel is the EN 14961 (in Slovakia, STN EN 14961 since 2010): Solid biofuels, specifications and categories of fuel, consisting of the following parts:

- Part 1: General requirements (EN 14961-1)

- Part 2: Wood pellets for industrial use (EN 14961-2)

- Part 3: Wood briquettes for non-industrial use (EN 14961-3)

- Part 4: Wood chips for non-industrial use (EN 14961-4)

- Part 5: Wood for burning in non-industrial uses (EN 14961-5)

- Part 6: Non-wood pellets for non-industrial use (EN 14961-6)

The goal of these European Standards is to provide definite and clear principles for the classification of solid biofuels, and these standards currently serve as a tool allowing the effective trade of biofuel because the conditions for quality are clear between the seller and buyer. This European Standard is prepared for all groups of users. The subject of this Technical Standard is the specification of categories for quality. The fundamental goal is to describe solid biofuels according to (STN EN 14961-1):

- origin and source

- main forms of trade 
Solid biofuels are traded in various shapes and sizes. The size and shape affect the manipulation of the fuel as well as the combustion process.

STN EN 14961 includes the physical-mechanical indicators of quality as:

1. Bulk density (STN EN 15103)

2. Particle size distribution (STN EN 15149)

3. Particle density (STN EN 15150)

4. Mechanical durability (STN EN 15210)

5. Length and diameter of the briquette (STN EN 16127)

The same standard defines the chemical-thermal indicators of quality as:

1. Moisture content (STN EN 14774)

2. Ash content (STN EN 14775)

3. Net caloric value (STN EN 14918)

4. Carbon, hydrogen, and nitrogen content (STN EN 15104)

5. Chloride, sodium, and potassium content dissolved in water(STN EN 15105)

6. Sulfur and chlorine content (STN EN 15289)

7. Volatility dust matter (STN EN 15148)

8. Content of primary elements (STN EN 15290)

9. Content of secondary elements (STN EN 15297)

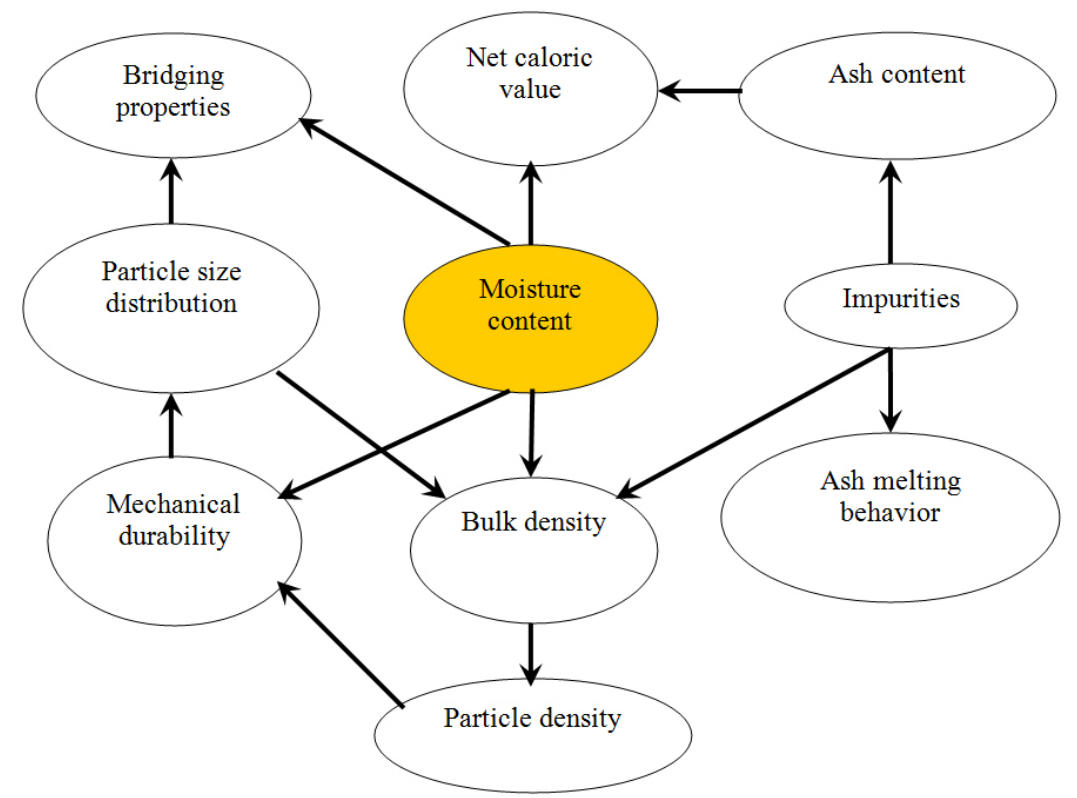

Figure 2.25: Dependence between the mechanical and chemical-thermal properties (Rönnbäck \& et al., 2012). 
In the following text, only a few monitored parameters, mainly mechanical ones, will be discussed. In terms of the proposed experimental research, it is not necessary or practical to describe the chemical-thermal indicators of quality. From experience, quality of the briquette is primarily evaluated according to its density. Below, some procedures are given for determining parameters.

Fundamentally, we know of several mechanical indicators. In practice, various procedures for determining some mechanical indicators of quality are distinguished. Amongst the most basic and mandatory indicators are those given by the set of Standards "CEN-TC 335/Solid biofuels", which describe bulk density, size of particles, density of particles, mechanical durability, and the length and diameter of the briquette.

Then, the mechanical indicators and procedures, which were used before EU Standards (Ö-NORM M 7135 a DIN 51731) for briquette density (DIN 51731), bulk density (SS 187120),or mechanical durability (SS 187120) are known. Some producers that own testing equipment to determine the afore mentioned standards, still test the basic parameters according to the "old" standards.

In practice, the procedures for simple determination of briquette quality are performed by submersion tests, visual evaluation, and the informative observation of hardness and strength (simple compression splitting).

According to valid European Technical Standards, quality is determined by the parameters in the following Tab. 2.7.

Table 2.7: Limit values for the indication of quality for pellets according to STN EN 14961-2.

\begin{tabular}{|c|c|c|c|c|c|}
\hline Indicator & Unit & Label & ENplus-A1 & ENplus-A2 & EN-B \\
\hline \multirow[t]{2}{*}{ Diameter ${ }^{1)}$} & $\mathrm{mm}$ & D06 & & $6 \pm 1$ & \\
\hline & & D08 & & $8 \pm 1$ & \\
\hline Length ${ }^{2)}$ & $\mathrm{mm}$ & $\mathrm{L}$ & & $3.15 \leq L \leq 40$ & \\
\hline Mechanical resistance & $w-\%{ }^{3)}$ & DU & $\geq 97.5$ & $\geq 97.5$ & $\geq 95.5$ \\
\hline Bulk weight & $\mathrm{kg} \cdot \mathrm{m}^{-3}$ & BD & & $\geq 600$ & \\
\hline Particle size (<3.15 mm) & $w-\%{ }^{3)}$ & $\mathrm{F}$ & & $<1$ & \\
\hline
\end{tabular}


Table 2.8: Limit values for indication of quality for briquettes according to STN EN 14961-3.

\begin{tabular}{llllll}
\hline Indicator & Unit & Label & A1 & A2 & B \\
\hline Diameter $^{1)}$ or & $\mathrm{mm}$ & $\mathrm{D}$ & to be est. & diameter, width & and length \\
Length $^{1)}$ & $\mathrm{mm}$ & $\mathrm{L}_{1}$ & & & \\
Width $^{1)}$ & $\mathrm{mm}$ & $\mathrm{L}_{2}$ & to be est. & diameter, width & and length \\
Height $^{1)}$ & $\mathrm{mm}$ & $\mathrm{L}_{3}$ & to be est. & diameter, width & and length \\
Mechanical resistance $^{2)}$ & $\mathrm{W}-\%^{3)}$ & $\mathrm{DU}$ & $\geq 95$ & $\geq 90$ & $\geq 90$ \\
Particle density $^{\text {g. }}$ & g.cm & DE & $\geq 1.0$ & $\geq 1.0$ & $\geq 0.9$ \\
\hline
\end{tabular}

a) $(\mathrm{m} / \mathrm{m})$ - weight ratio.

${ }^{1)}$ actual diameter of briquette; ${ }^{2)}$ informative test only if they are traded in bulk; ${ }^{3)}$ in the delivered state, $\%(\mathrm{~mm})$ - weight ratio in percentage.

In determining the briquette quality according to STN EN 14961-3, the briquette density is investigated instead of the bulk density. Furthermore, the length, diameter, and mechanical durability of the briquette are monitored. The European STN EN 14961-3 Standard defines briquettes made from wood in three quality categories: A1, A2, and B. This division depends on the properties and source of the raw material as well as the characteristics and parameters of the product.

\section{Density of the briquette and bulk density}

Briquette density is the most important indicator of quality. The density for briquettes made from wood (HP1) given by ŐNORM M 7135 must be greater than $1.0 \mathrm{~kg} . \mathrm{dm}^{-3}$. Briquettes from core wood (RP1) must have a density of at least $1.0 \mathrm{~kg} . \mathrm{dm}^{-3}$. The German DIN 51731 Standard states that briquettes must have densities between 1.0 $1.4 \mathrm{~kg} . \mathrm{dm}^{-3}$, whereas the certification scheme of DIN Plus requires a minimum value of $1.12 \mathrm{~kg} . \mathrm{dm}^{-3}$. There is an obvious and direct dependence between the density of the briquette and bulk density. The Swedish SS 187120 Standard (for pellets made from wood) does not define a required density for the briquette but rather the minimum bulk density in three categories. The Austrian ŐNORM M 7135 and German DIN 51731 a DIN Plus do not define requirements for the bulk density of the briquette/pellets. Density of the product is important for its manipulation. Briquettes must be cohesive so as not to crack and break into small pieces. Higher briquette densities from wood matter typically increase their strength. Briquettes with higher density also have a longer burn, which is a very important factor considering their use as fuel. Higher density also positively affects the long-term stability of the briquette volume and shape and decreases its ability to absorb moisture.

DIN 52182 (supplement to Standard DIN 51731) describes the procedure for evaluating briquette density. After densification, the briquette is placed in a stable climate. Gradually, the diameter, length, and weight are measured; however, these initial values are not possible to interpret due to dilation, a common undesirable 
effect. It is necessary however to consider dilation when determining the density. Dilation changes the dimensions of the briquette due to relaxing effects of the pressing pressure and depending on the mutual interactions of the pressing temperature, moisture of the input material, and input fraction size. Based on these values, dilation character can be positive or negative (the size of briquette can increase or decrease). The mentioned Standards also take into consideration the stabilization duration, which is the time required for the briquette to stabilize and dilate. During this time, the briquette is continually measured. If, within 24 hours, the weight changes by $0.1 \%$ or less, the briquette is considered stable. The final weight is then defined as the weight of the briquette $m_{n}(\mathrm{~kg} ; \mathrm{g})$. At this point, the geometric dimensions are measured, the volume is calculated $V_{n}\left(\mathrm{~m}^{3} ; \mathrm{cm}^{3}\right)$, and the specific density is determined from Equation2.12. The density must be specified within $0.01 \mathrm{~g} . \mathrm{cm}^{-3}$.

$$
\rho_{n}=\frac{m_{n}}{V_{n}}\left(\mathrm{~g} \cdot \mathrm{cm}^{-3}\right)
$$

Bulk density is defined as the ratio of weight $m_{v}$ and volume $V_{S^{*}}$

$$
\rho_{S}=\frac{m_{v}}{V_{S}}\left({\left.\mathrm{~g} \cdot \mathrm{cm}^{-3}\right)}^{-1}\right.
$$

\section{Briquette/pellets abrasion and mechanical durability}

Many methods exist today to specify the mechanical and abrasion resistance of the pellets. The method used depends on the Standard used. The abrasion and mechanical durability of the pellets is understood as the resistance to crumbling or chaffing as a result of manipulation, storage, and transport. Some Standards describe procedures to determine the amount of chaffing, whereas others determine the mechanical durability. Testing is done by shaking and rubbing the pellets. However, a pellet is never tested for both abrasion and mechanical durability. It can be said that the abrasion value after testing is the "opposite" of the value for mechanical durability. For example, from $100 \%$ of the tested volume, if $2.5 \%$ is rubbed from the pellets after abrasion testing, then $2.5 \%$ is wasted due to abrasion. In terms of mechanical durability, after testing, $97.5 \%$ would be the recorded testing value, meaning that $97.5 \%$ of the tested pellets remain intact.

Abrasion is commonly defined for pellets only (HP4 and HP5 for DIN 51731 and DIN Plus Standards; and HP1, HP2, RP1, and RP2 for the ŐNORM M 7135 Standards). Abrasion is an important parameter for the practical assessment of quality. These Standards prescribe its required value and the procedure to determine it. Abrasion is related to requirements that restrict the formation of dust particles in the automated transportation of solid biofuels and thus is an indicator of the reduced risk of explosion during burning. 
Measuring abrasion for large-diameter briquettes is typically not done; however, STN 441309 Standard specifies the "Determination of briquette abrasion".

Abrasion testing is simple and quick. The test is performed using the Lignotester machine (LT - II or NHP 100), which is shown in Fig. 2.26 on the left. Pellets are tested without any dust particles; thus, before the test, a fine sieve is used to remove any dust from the surface of the pellet (3.15mm mesh in regards to ISO 3310-1). After the preliminary dust removal, $100 \mathrm{~g} \pm 0.5 \mathrm{~g}$ of pellets are placed in the testing apparatus (Ligno tester) and subjected to $70 \mathrm{mbar}$ of airflow over a 60 -second interval. The artificial airflow simulates transport, collision, and friction between individual pellets and the walls of the apparatus. Abrasion is then specified as the weight of left-over material in a percentage $\%$ according to Equation 2.14. This test is performed five times, and the recorded abrasion value is specified as the arithmetic average of the values obtained from each test.

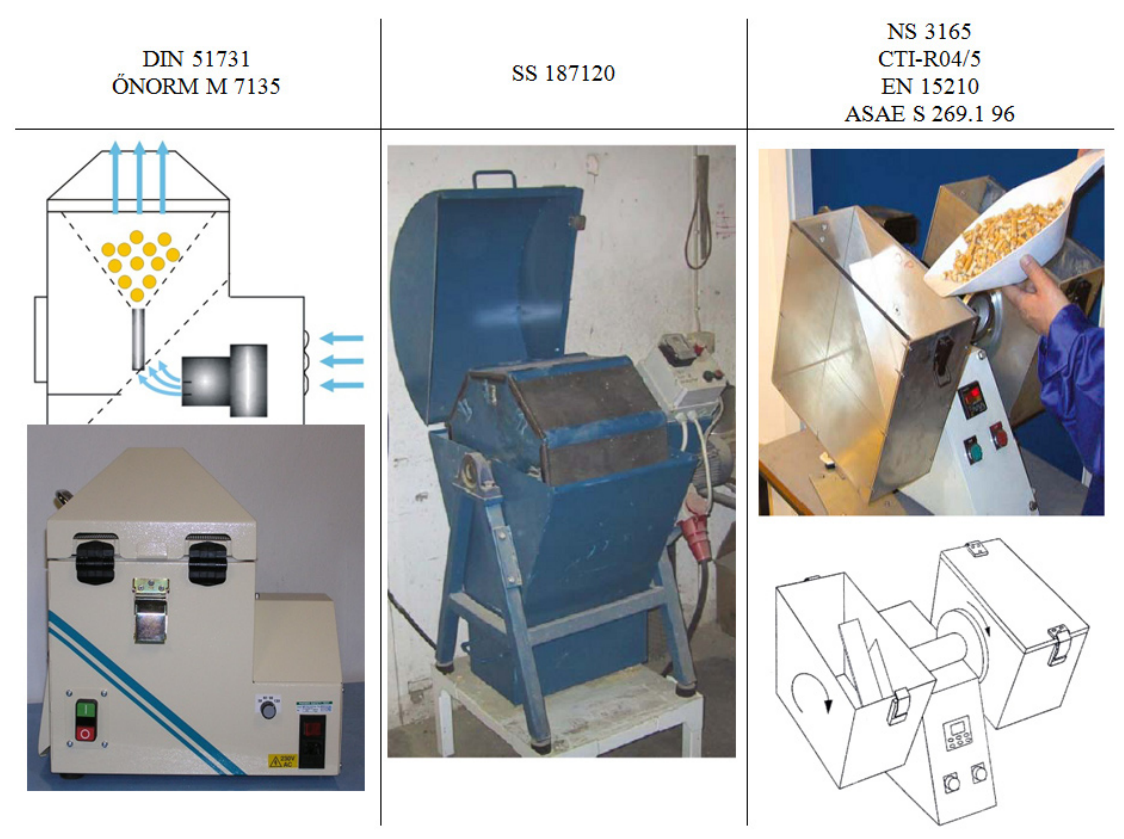

Figure 2.26: Apparatus used for testing the mechanical resistance depending on standards used.

Abrasion calculation:

$$
A R=\frac{m_{E}-m_{A}}{m_{E}} .100
$$

where $A R-$ abrasion (\%),

$m_{E}$ - weight of pellets before test $(\mathrm{kg})$,

$m_{A}-$ weight of pellets after test $(\mathrm{kg})$. 
Acceptable deviations for the arithmetic average is within $0.2 \%$ of the maximum allowable abrasion with respect to ŐNORM M 7135 (DIN Plus $\mathrm{AR}_{\max }=2.3 \%$ ).

The mechanical durability, or mechanical strength, is the ability for each compressed biofuel particle (briquette/pellet) to remain in an undisturbed state, for example, during manipulation and transport. Mechanical durability is an important parameter for the practical assessment of quality. This parameter is related to the requirement to reduce dust in the automated transportation process to prevent explosion when burning.

To evaluate the mechanical durability, a test-appropriate dosage of pellets is shaken in a testing chamber. The testing apparatus can contain up to three of these chambers. Mechanical durability is calculated from the weight of left-over samples and the weight of separated material after testing. The minimum sample size is $2.5 \mathrm{~kg}$. Samples should be divided into 4 equal dosages, which are sieved according to ISO 3310-2. Dosages are tested after an initial separation of dust particles. Determining the mechanical durability requires at least two tests of sieved pellets with a dosage weight of $500 \pm 10 \mathrm{~g}$. The sample must be weighed before testing to within $0.1 \mathrm{~g}$ of accuracy. In the testing apparatus, the pellets are chaffed by means of rotational movement in the chamber. After 500 rotations at $50 \pm 2 \mathrm{~min}^{-1}$, the pellets are removed, sieved by hand, and weighed. The mechanical resistance is then calculated using Equation 2.15. The results must be calculated to the nearest hundredth of a gram $(\mathrm{g})$.

$$
D_{U}=\frac{m_{A}}{m_{E}} \cdot 100
$$

where:

$D_{U}$ - mechanical resistance (\%),

$m_{A}$ - weight before sieving and testing $(\mathrm{kg}, \mathrm{g})$,

$m_{E}$ - weight after sieving and testing $(\mathrm{kg}, \mathrm{g})$.

\section{Strength in compression}

Strength in compression is another important indicator of quality with respect to resistance when manipulating, transporting, and storing briquettes. Standards so far do not specify any required value for this indicator/parameter. Therefore, stating the strength value in compression is based on the STN 441309 Standard for the "Collection and modification of mechanical testing of carbon based briquettes." 

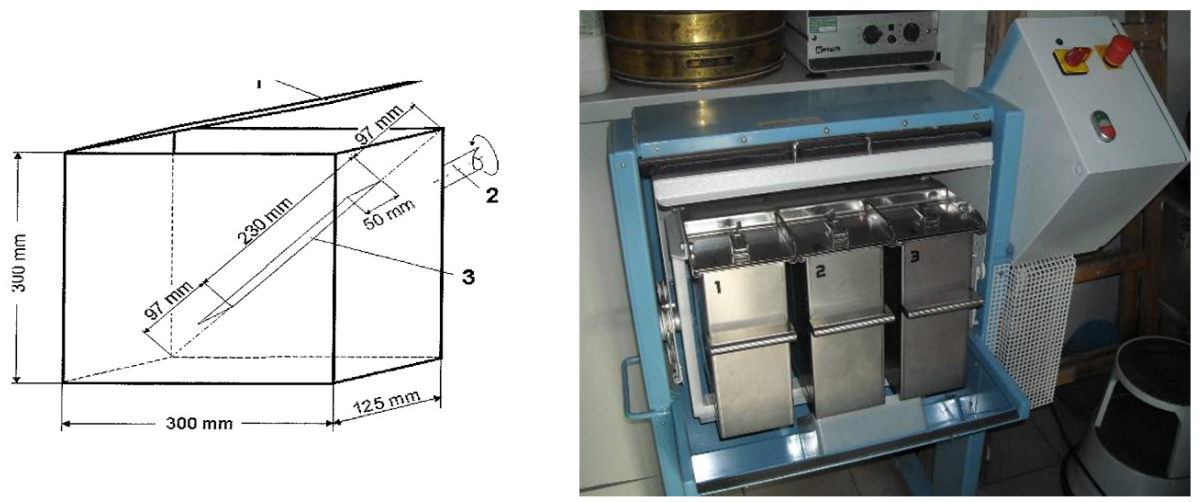

Figure 2.27: Apparatus for testing the mechanical resistance of pellets/briquettes STN EN 15210-1. (1-filling cavity, 2-driven shaft, 3-contact plate).

The term "briquette strength" refers to the maximum pressure on the punch or piston during compression testing under the following conditions according to the following expression:

$$
\sigma_{B t}=\max p(\mathrm{MPa})
$$

The briquette is placed for testing between the circular pistons of the press, where it is uniformly loaded with increasing pressure until complete disintegration of the briquette. The test utilizes undisturbed and complete briquettes. Briquettes are placed in the center between two circular pistons of $30 \mathrm{~mm}$ diameter. The pressing piston uniformly increases load on the briquette throughout the test, and the maximum pressure obtained during the test before disintegration gives the strength of the briquette in compression. Seven randomly-selected briquettes are used for testing, and the recorded value from the tests is determined from the arithmetic average of five test values (the highest and lowest test values are excluded). The resulting strength of the briquette is then:

$$
\sigma_{B t}=\frac{1}{5} \cdot \sum_{i=1}^{5} p_{i}(\mathrm{MPa})
$$

where: $\sigma_{B t}-$ strength of briquette in compression (MPa),

$p_{i}$ - value of the maximum pressure at the $i$-th measurement (MPa).

This standard is valid for carbon-based briquettes; however, it can also be applied to briquettes made from biomass.

According to STN 441309, two pressure-related strength tests for cylindrical briquettes exist. They are a test for simple compression and a splitting test. For both tests, the force is measured until the specimen breaks apart (failure). The indicator 
value for the splitting test is proportional to the force and inversely proportional to the length of briquette.

The strength test by splitting is conducted according to STN 441309. Strength test of a cylindrical briquette is calculated by:

$$
\sigma_{B r}=\frac{F_{\text {max }}}{L_{N}} \quad\left(\mathrm{~N} \mathrm{~mm}^{-1}\right)
$$

where: $\sigma_{B r}$ - strength of briquette when splitting $\left(\mathrm{N} . \mathrm{mm}^{-1}\right)$,

$F_{\text {max }}-$ maximum force acting on the briquette $(\mathrm{N})$, $L_{N}$ - length of briquette $(\mathrm{mm})$.

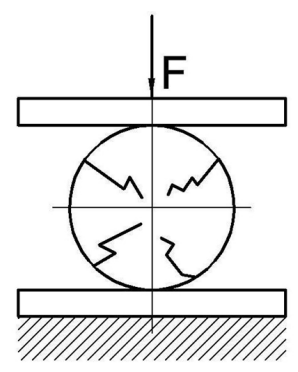

Figure 2.28: Splitting test.

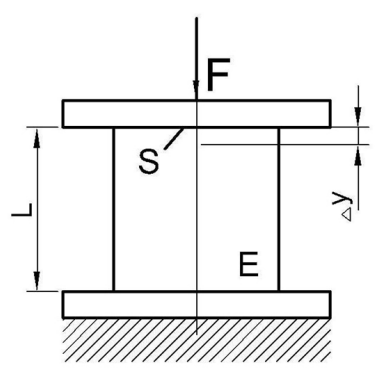

Figure 2.29: Strength test in compression.

Strength test of briquette in simple compression is performed according to STN 441309. 
Strength of a cylindrical briquette is calculated as:

$$
\begin{gathered}
\sigma_{B t}=E . \varepsilon \quad(\mathrm{MPa}) \\
\varepsilon=\frac{\Delta y}{l_{n}} \quad(-)
\end{gathered}
$$

where:

$\sigma_{B t}$ - is the strength of the cylindrical briquette ( $\left.\mathrm{MPa}\right)$,

$E$ - module of elasticity (MPa),

$\varepsilon$ - ratio of deformation (-),

$\Delta y$ - pressing the briquette after achieving maximum loading force (mm),

$l_{n}$ - length of briquette $(\mathrm{mm})$.

\section{Determining quality of pellet - practical procedures}

Apart from the above description of the basic Standards defining the indicators of quality, it is possible to use some practical procedures to obtain some initial information about the briquette. However, these procedures are not supported by any standards. Biofuel producers and sellers only use them for simple and quick evaluation of briquette quality.

\section{Pellets/Briquette colour}

Colour is usually the first property of pellets that a buyer first notices. Colour usually indicates the type of material used (wood, straw, pulp, etc.). However, colour can differ between pellets of the same material, indicating the method of compaction, finishing, quality of raw material, and cleanliness. In general, "light" colour pellets are considered high-quality. They typically have low ash content and are usually made from a clean and high-quality raw material. "Dark" pellets usually contain wood bark, which has a porous structure; as a result, chemical substances and dust particles are easily caught in these pores. Dark pellets also indicate a high degree of sawdust degradation or the use of raw material from old wood chips (Mikuláš\&Vícha, 2006; 2007). In some countries, the colour is a very important quality for buyers, and therefore dark product is undesirable in a supplier's point of view. Based on the above, and proven by experience, darker briquettes typically contain an undesirable composition compared to light briquettes. 


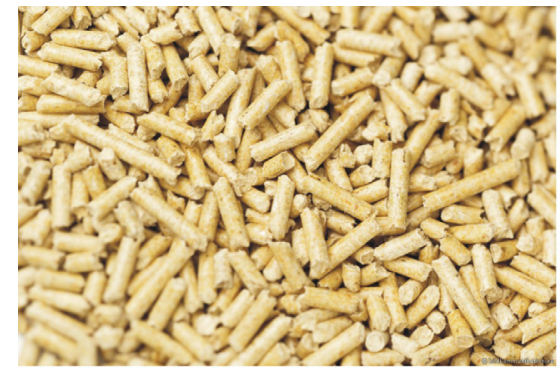

Pellets made from spruce sawdust

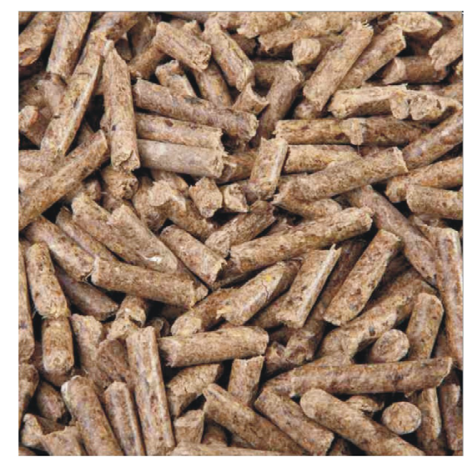

Pellets from mixed material (spruce and beech)

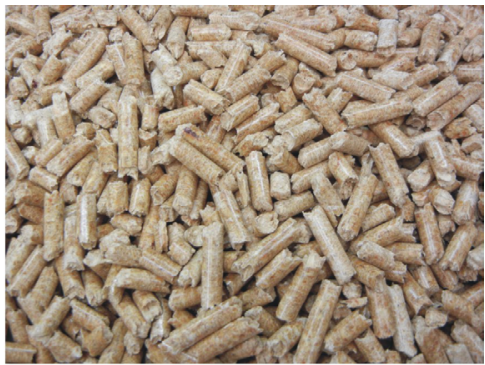

Pellets made from beech sawdust

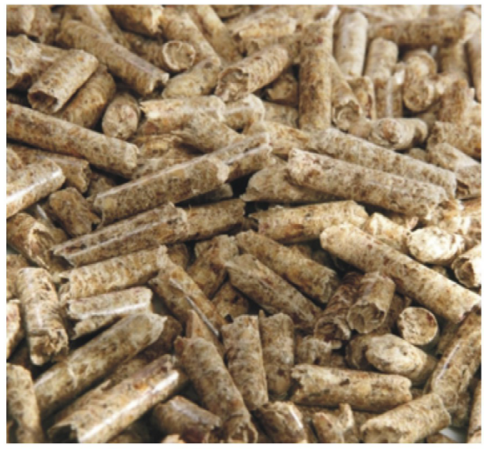

Pellets made from oak sawdust

Figure 2.30: Various colours of pellets depending on the raw material used.

The effectiveness of certain wood types in the burning process is generally known. Pellets made from spruce or pine typically do not cause any problems in the burning process itself. However, pines emit higher CO emission for its production (when storing) and has an undesirable smell. These raw materials have high sap content, and, when burning, there is a tendency for debris and carbon to form in the chimneys of the furnaces. Spruce and other hard woods have very good burning characteristics in furnaces with low emissions, light aroma, and low sap content.

It is necessary to comment that darker pellets are not only caused by wood bark content and older material, but also by the production process. Since the production process of pellets inherently uses high temperatures and pressures, low moisture content of input fractions, and slower pressing process, pellets without any bark wood can also turn out darker. A drawback to pressing can be a loss in volatile elements, which degreases the product heat potential. Pressing at higher pressures causes the outer surface of the pellet to sinter. This effect is actually a benefit as cracks do not form on the surface of the pellets. The pellets are also more compact and coherent. If the pellets as a whole are darker but without any dark spots, it can be assumed that there is no bark wood in the pellet material. 


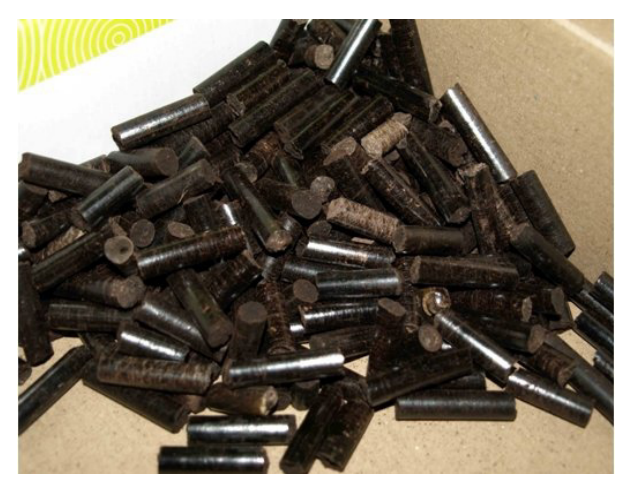

Figure 2.31: Pellets made from core wood.

\section{Surface of the briquettes/pellets}

The surface of the briquette is another significant indicator of quality. A shiny, compact surface indicates high quality. If the surface is spotty and non-uniform, it can indicate in homogeneity in the production process and thus a non-uniform burning characteristic in the furnace (Mikuláš \& Vícha, 2006; 2007).

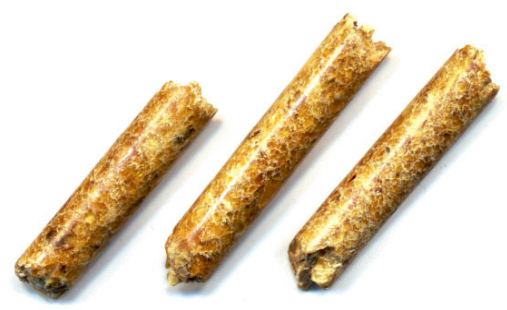

Figure 2.32: Shiny, uniform surface of the pellet.

Briquettes/ pellets hardness

The hardness of the briquette typically relates to quality. In principle, harder briquettes have higher quality and thus higher concentration of energy (greater heat potential in the same volume). Hardness can be determined by the so-called submersion test

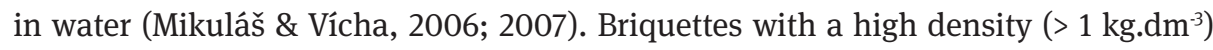
must be submerged. If the briquette crumbles within 5 minutes, the product is of very low quality. If the briquette crumbles within 15 minutes, the product is of average quality. If the briquette lasts up to 20 minutes without crumbling, the product is of high quality. 


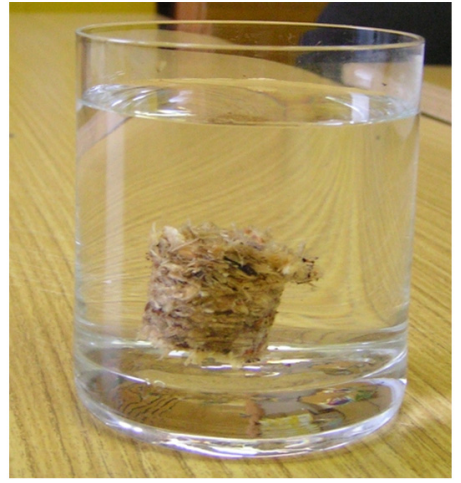

Low quality briquette

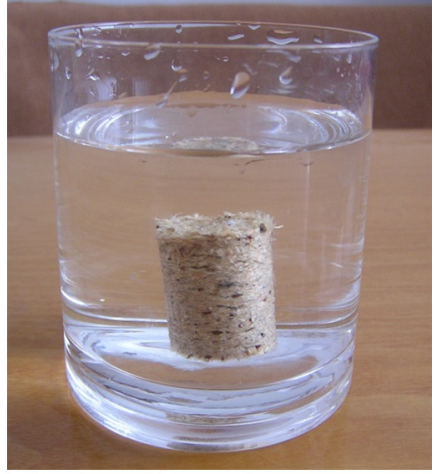

Average quality briquette

Figure 2.33: Hardness testing by submersion into water.

Another way to test the product hardness is to use a specialized apparatus (KAHL). A briquette is placed between a die and punch. The apparatus applies force on the briquette until it breaks.
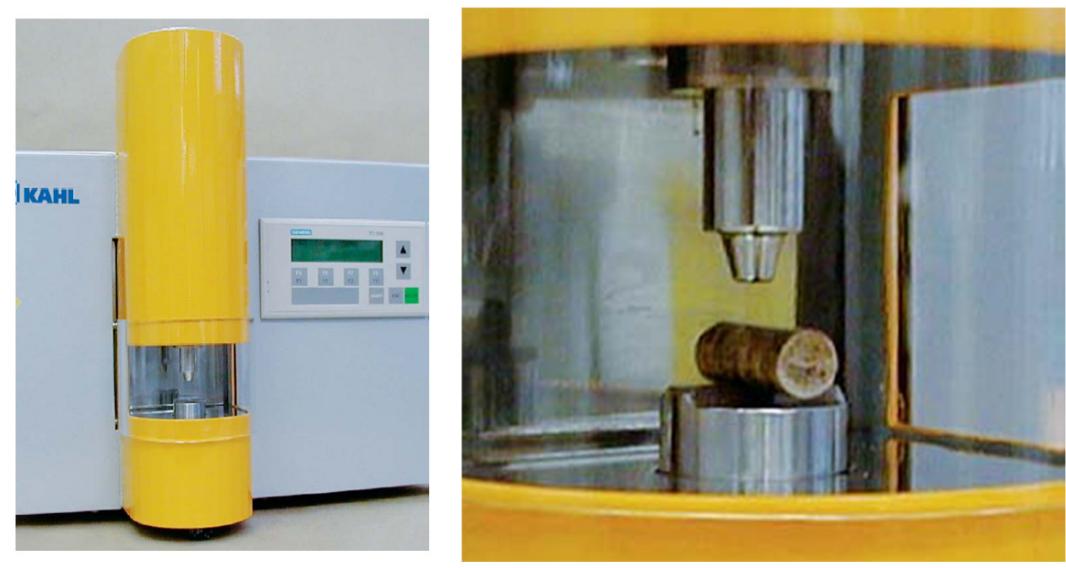

Figure 2.34: KAHL apparatus for testing pellet hardness.

\subsection{Parameters Affecting the Resulting Quality of the Briquette}

The goal of experimental research described within this monograph is to define the effects of some parameters on the resulting quality of briquette and to determine the relations between these parameters. After a detailed analysis of the known affecting parameters, the effects on the densification process can be observed. The analyses of 
these parameters were divided into three groups since they are not of similar character. The first group of parameters relates to the type of pressed material; the second group relates to the pressing technology; the third group envelopes all structural parameters:

1. pressed material:

- type,

- fraction moisture,

- moisture content,

- temperature,

2. pressing parameters:

- pressing method,

- temperature in the pressing chamber,

- pressure in the pressing chamber,

- pressing speed,

- holding time,

3. structural parameters:

- diameter, length, and shape (conical) of the pressing chamber,

- type, diameter, and shape of the pressing tool,

- material, finishing (surface roughness), and surface treatment of the pressing chamber and tool,

- back-pressure acting on the compressed plug,

- length of the cooling channel,

Obviously, it is not possible to perform experiments for all of the above parameters, and therefore it was necessary for the present study to choose the most appropriate parameters. Parameters which showed the greatest effect on the resulting quality of the briquette and were also easily measured were chosen. Based on these criteria, one material was chosen from the first group of parameters with definite moisture content and fraction size for analysis. From the pressing parameters, based on detailed analysis, the pressing pressure and temperature in the pressing chamber were chosen.

\subsubsection{Type of Material Being Pressed}

The type of material chosen for pressing is a very important factor that can significantly affect the final quality of the briquette. If wood waste is pressed, the parameters (pressure, temperature, etc.) are different than those of other types of raw materials. At the same time, pressing soft wood is different than pressing hard wood. For different types of materials and input conditions, different briquette properties (heating potential, moisture content, ash content, etc.) are obtained. Each type of material has a specific density. The density significantly impacts the mechanicalphysical properties of wood. A higher specific density of the input material generally 
results in better densification. Therefore, we can say that the density of wood affects its mechanical and chemical processing, influencing the quantity of wood mass required. For example, hard wood is stronger, harder and more resistant to wear and mechanical processing than soft wood.

Table 2.9: Density of the most common industrial wood materials (Bozděch \& Černák, 1987; Kremer, 1995).

\begin{tabular}{ll}
\hline Wood species & Density of absolutely dry wood $\rho_{0}\left(\mathrm{~kg} \cdot \mathrm{m}^{-3}\right)$ \\
\hline Spruce, fir, poplar & $350-400$ \\
Basswood, aspen & $400-450$ \\
Pine & 500 \\
Larch, Douglas fir, willow, alder, birch & $500-600$ \\
Ash, maple, chestnut & 630 \\
Brest, oak & $640-650$ \\
Beech & 680 \\
Acacia, hornbeam & $730-800$ \\
\hline
\end{tabular}

Another factor affecting material densification is the presence of various types of dirt, dust, and cork, which negatively effects the briquette strength. Heating potential of the material is also very important since the densified product will concentrate this energy uniformly.

Porosity relates to density in wood. Porosity is the volume of the pores in a unit volume of wood. Pores are cavities created by the lumen, the intercellular space with capillary radius greater than $100 \mu \mathrm{m}$, and cavities in the cell walls of each individual wood element up to $100 \mu \mathrm{m}$.

The density of wood and its weight are mainly affected by the moisture content, thickness of annual growth rings and ratio of summer wood, position in the trunk, and tree life. Wood density and weight increases with water content such that the weight and volume of the wood do not change proportionally. Whereas the weight of wood can increase with rising moisture content until its complete saturation, the volume of the wood can change until the PCS. Further increase in moisture within the wood does not change its volume.

From data shown in the following figure, it is obvious that the type of material being pressed significantly affects the pressing process. To be consistent, it is necessary to work with one type of material. Due to time limitations, the experiments in the first phase were performed for one type of wood material. According to the procedures presented in this work, it should be possible to repeat the experiments with different types of materials. 
Based on available sources of wood sawdust, pine sawdust was utilized in the first phase of the experiments. Pine (pinus sylvestris L.) is a coniferous tree with a triangular (conical) shape and originates from central and northern Europe, Asia, and Siberia. Pine wood is yellow or pinkish with a light brown structure when freshly split and turns reddish brown when exposed to the air. Pine is less resistant to biological disease, but is easily dried and processed. It has a wide application in construction materials (interior, exterior, and even marine), in furniture, and in chemical industries (pulp, wood fibre, turpentine) (Kremer, 1995). Some mechanical and thermal properties of pine are given in the following table.

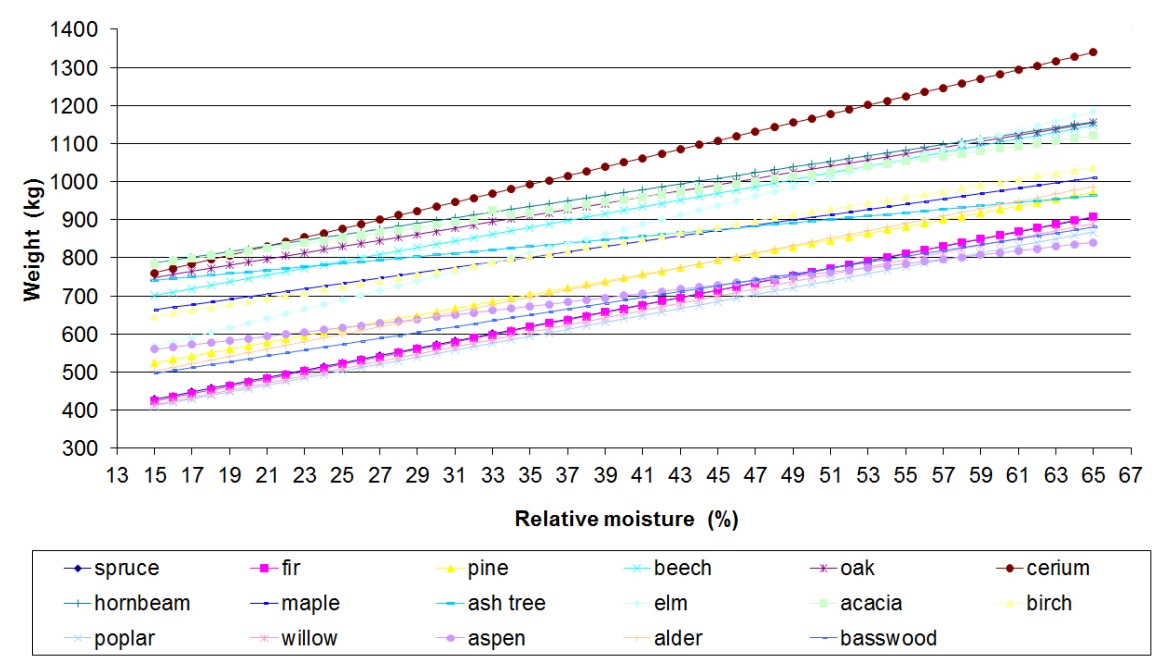

Figure 2.35: Dependence of wood weight on the moisture of different types of wood (Bozděch \& Černák, 1987; Kremer, 1995).

\subsubsection{Pressure in the Pressing Chamber}

The pressing pressure is one of the most important factors affecting the resulting briquette strength. With increasing pressure, the briquette tends to strengthen until the yield strength of the material is reached. This also reduces the tendency of the briquette to absorb moisture when stored (Horníková, 2009). In the densification of organic waste, 55 to $355 \mathrm{MPa}$ of pressure is typically used (Kegel, 1948). It is important to find an ideal combination of force and force vector direction to obtain a highly compact briquette. The pressing method has an effect on the composition of the briquette layers and thus on its overall strength. 
Table 2.10: Some selected properties of pine - pinus silvestris (Holm \& et al., 2006; Chovanec \& et al., 2006; Požgaj \& et al., 2006).

\begin{tabular}{|c|c|c|c|c|}
\hline Density & $\begin{array}{l}\text { Volumetric } \\
\text { weight }\end{array}$ & \multirow[t]{2}{*}{ Unit weight when dry } & \multirow{2}{*}{$\begin{array}{l}\text { Volumetric } \\
\text { shrinkage }\end{array}$} & \multirow{3}{*}{$\begin{array}{l}\text { Heating } \\
\text { potential at } \\
\mathbf{w}_{\mathrm{r}}=25 \% \\
13,6 \mathrm{MJ.kg}\end{array}$} \\
\hline at $w_{r}=0 \%$ & at $w_{r}=25 \%$ & & & \\
\hline $500 \mathrm{~kg} \cdot \mathrm{dm}^{-3}$ & 680 kg.pm & $510 \mathrm{~kg} \cdot \mathrm{m}^{-3}$ & $11,2-12,4 \%$ & \\
\hline $\begin{array}{l}\text { Strength in } \\
\text { compression }\end{array}$ & $\begin{array}{l}\text { Bending } \\
\text { strength }\end{array}$ & \multirow{2}{*}{$\begin{array}{l}\text { Module of elasticity } \\
\text { in the direction of } \\
\text { fibres } E_{L}\end{array}$} & \multirow{2}{*}{$\begin{array}{l}\text { Module of elasticity } \\
\text { in the radial } \\
\text { direction of fibres } E_{R}\end{array}$} & \\
\hline \multirow[t]{2}{*}{55 N.mm-2 } & $80 \mathrm{~N} \cdot \mathrm{mm}^{-2}$ & & & \\
\hline & $\begin{array}{l}\text { Bending } \\
\text { strength }\end{array}$ & $6634 \mathrm{MPa}$ & $320 \mathrm{MPa}$ & \\
\hline Hardness & at $w_{r}=12 \%$ & Poissons number $v_{L R}$ & Poissons number $\mathrm{v}_{\mathrm{RL}}$ & \\
\hline $28,5 \mathrm{MPa}$ & $1900 \mathrm{~N}$ & 0,337 & 0,016 & \\
\hline
\end{tabular}

The analysis of pressure is very interesting and complicated. To obtain a high quality briquette, it is necessary to ensure a maximum pressure within the pressing chamber. This value represents the pressure needed for compaction and feed of the briquette through the chamber. Other parameters affect the pressing pressure. Biomass under pressure undergoes two types of deformation: elastic and plastic (Chuchrjanskij \& Lehký, 1953). Elastic deformation returns to its original position once the pressure is released; however, plastic deformation permanently deforms the material. The pressing temperature, pressure, and moisture content of the material have a direct effect on the elastic and plastic deformation observed. In Figure 2.36, obvious changes can be seen in pressure when pressing a pine briquette with consistent fraction size of input material $(L=2 \mathrm{~mm})$, moisture content $\left(w_{r}=10 \%\right)$, and temperature $\left(55^{\circ} \mathrm{C}\right)$. However, the pressing pressure was different in each test. We can see that lower pressures resulted in crumbling of the briquette. At higher pressures, the briquettes are more consistent and compact. Fig 2.37 confirms the reasoning that higher pressures increase briquette density.

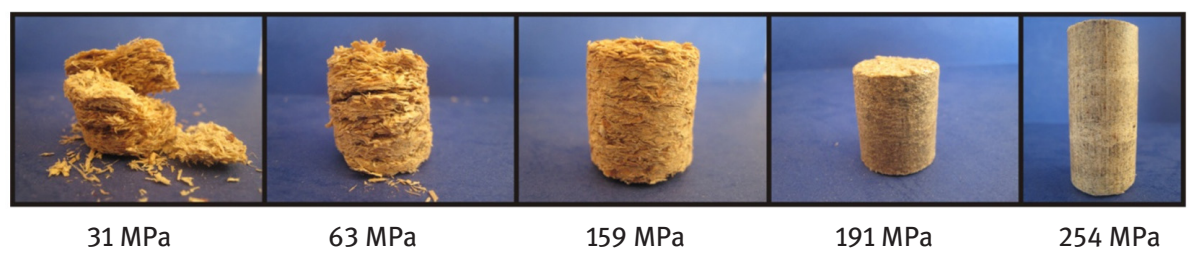

Figure 2.36: Briquettes with different acting pressures (Križan, Šooš \& Vukelić, 2008). 


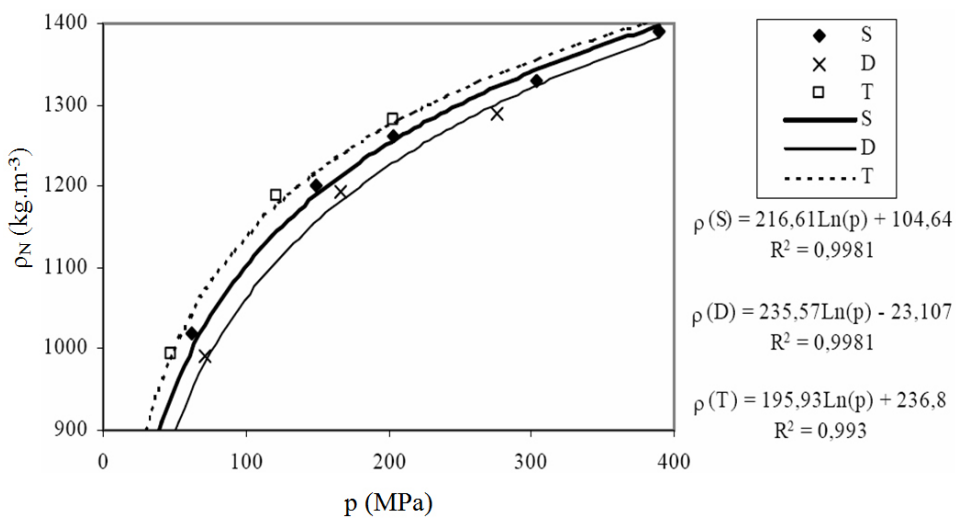

Figure 2.37: Dependence of density on the pressure for pellets made from pine (S), oak (D,) and reed (T). $\left(\mathrm{T}=20^{\circ} \mathrm{C}, \mathrm{w}_{\mathrm{r}} \approx 7 \%\right)($ Kegel, 1948).

\subsubsection{Pressing Temperature}

The pressing temperature has a significant effect on the quality and strength of the briquette. This parameter influences the excretion of lignin from the cellular structures. Lignin is a very important substance in the densification process because it confers adhesive effects in the pressed material. It connects fibres and acts as a reinforcing factor in the cellular molecules. The more lignin in the input material, and the more that can be extracted in the pressing process, the more uniformity and thus quality can be achieved from the resulting briquette. Lignin strengthens the briquette primarily in compression, but lignin is excreted only at certain pressing temperatures, which must be attained for the densification process to be optimized. Lignin plasticizes at approximately $120^{\circ} \mathrm{C}$, but this temperature, of course, depends on the exact material being pressed. From the known dependencies between strength and temperature, we know that it is not necessary to excessively increase temperature (Fig. 2.38). Higher temperatures not only evaporate water but also volatilize substances. Therefore, it is necessary to evaluate exactly how much the temperature should rise without losing heating potential of the briquette. Volatile substances are, by the way, very important components of a biofuel in terms of their heating potential.

Pressing at room temperature $\left(20^{\circ} \mathrm{C}\right)$ results in an unstable briquette; the product has low strength, which causes faster crumbling when burning, resulting in a faster burn. With constant pressure but increased temperature, the strength of the briquette increases until a certain point. Increasing the temperature from $20^{\circ} \mathrm{C}$ to $100^{\circ} \mathrm{C}$ yields insignificant losses from the evaporation of water (Holota \& et al., 1984). Heating to $150^{\circ} \mathrm{C}$ initiates the evaporation of volatile substances, which reduces the heating potential of the briquette (Holota \& et al., 1984). Of course, temperature also affects the moisture content of the product. 


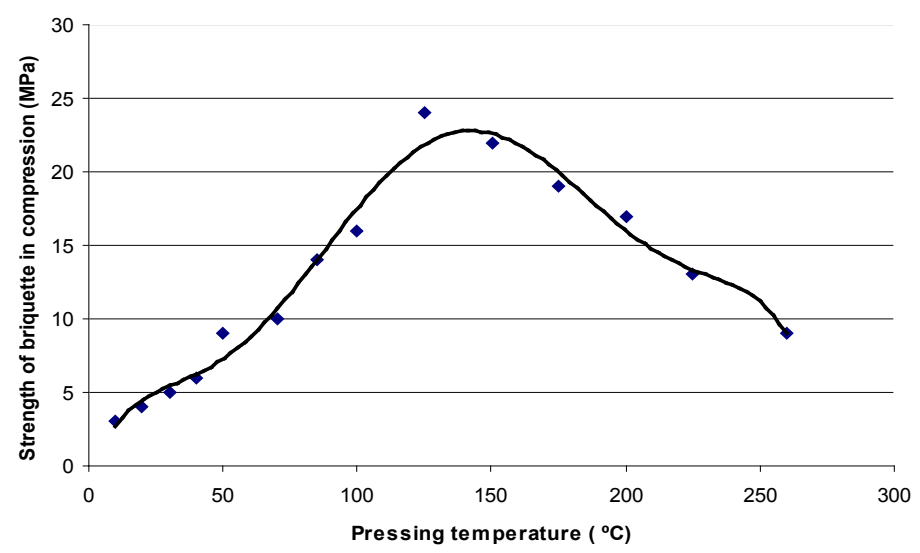

Figure 2.38: Dependence of briquette strength on the pressing temperature $\left(p=100 \mathrm{MPa}, \mathrm{w}_{\mathrm{r}}=20 \%\right.$, $\mathrm{L}=1 \mathrm{~mm})($ Sitzmann \& Buschhart, 2009).

When heating wood biomass, its physical and chemical properties change (Chuchrjanskij \& Lehký, 1953). One sign of this change is colour. High temperatures cause the vessels within the wood to decompose into their base elements. It is known that wood consists of cellulose, lignin, and hemicelluloses. Cellulose creates the primary structure, whereas the lignin and hemicelluloses influence this structure. Temperature and moisture content affectsubstances filling the cellulose structure by causing them to soften and plasticize. Heat alone can cause this to occur; however, this phenomenon is not nearly observed to the same extent when moisture is also an influence (Solár, 1997).

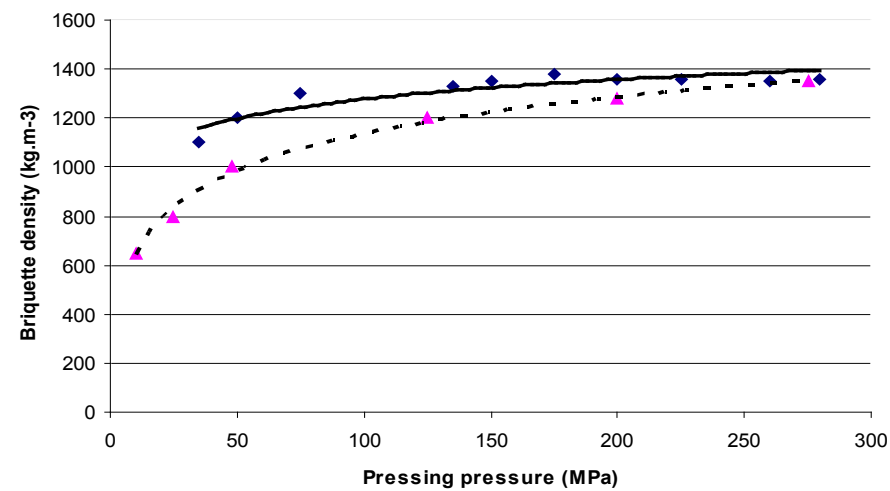

Figure 2.39: Dependence of density on pressure when pressing preheated samples at $\mathrm{T}=200{ }^{\circ} \mathrm{C}$ and room temperature $\mathrm{T}=20^{\circ} \mathrm{C}$ (Kádarová, 2005). 
Pressureand temperature go "hand in hand" in the densification process. If we increase the pressure, we can reduce the required temperature, and vice versa. This is nicely shown in Figure 2.39. Pressing at higher temperatures obtains the desired density without increasing the pressure. It is also generally known that it is economically advantageous to increase temperature rather than pressure.

Under the effects of heat and moisture, the physical-mechanical properties of wood biomass temporarily or permanently change. Temporary changes cause the material to return to its original state once cooled. These changes occur mainly when the pressing conditions are not optimal with respect to the pressed material. Temporary changes include the sharp increase in elastic deformation when heated and the resultant change in strength at different temperatures. Permanent changes in the properties of wood biomass, caused by the temporary increase in temperature, do not allow the material to return to its original state after cooling. Permanent changes occur under optimal pressing conditions in terms of the material properties of the pressed material. The effect of heat on the permanent physical-mechanical and chemical properties of wood biomass depends (to an extent) on the temperature, method used, and heating duration. Increasing temperature and prolonging the heating process increases these effects (Chuchrjanskij \& Lehký, 1953).

\subsubsection{Moisture of the Input Fraction}

The maximum moisture content of the material is an essential condition for the optimal densification of biomass. The effects of moisture content on the material must be known to produce quality briquettes as defined by Standards. It is known from specific results from research discussed in the literature that density and strength depend on the moisture content of the pressed material, for example, in the case of pressing wood sawdust (Kegel, 1948). It is also known that other materials, when pressed, those characteristics have similar dependencies. We can therefore say that the briquette quality depends on the moisture in the pressed material and that a large difference is not expected in the optimal level of moisture between different materials. The optimal value, however, is not known. Based on our knowledge and experience, an acceptable level of moisture is from $8-18 \%$ (Šooš, 2007). If the moisture content of the pressed material is very low, or very high for that matter, the particles become inconsistently arranged, and the resulting briquette becomes unstable. Research and experiments have shown that moisture has an effect on the plasticization of lignin. The temperature at which lignin softens depends also on the type of wood and method of isolation. The temperature at which lignin changes phase from a solid to a plasticized state is proportional to its molecular mass and non-proportional to the level of moisture (Hejma\& et al., 1981). The effects of water content (material moisture) on the transition temperature of lignin can be seen in the following figure. 


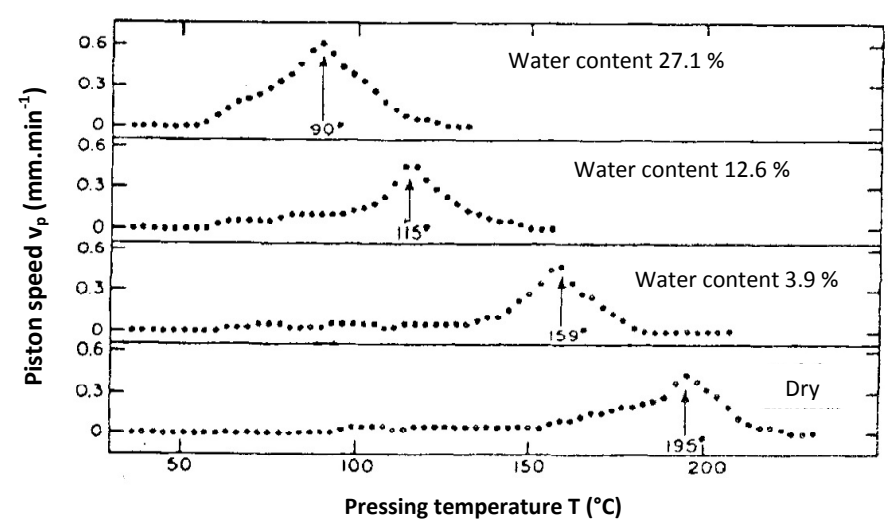

Figure 2.40: Effects of moisture on the transition temperature of spruce lignin at different piston speeds (Hejma \& et al., 1981).

The mutualinteraction between the material moisture, pressing pressure, and pressing temperature is very interesting. If we set the process to the optimal temperature and pressure but allow moisture content to be out of the optimal range, the resultant briquette will be unstable. Proper temperature ensures that the lignin plasticizes in the pressed material, and the optimal pressure provides densification of this material. However, if the pressed material has high moisture content, escaping water in the form of steam cracks the briquette. If the moisture content is very low (below $6 \%$ ), the briquette must be pressed with a higher temperature and pressure to obtain a highquality product, which becomes economically unprofitable. With reduced moisture content, the heating potential must be increased. A certain moisture level causes a higher temperature in the densification process and thus a higher quality briquette. Optimal moisture level improves the compaction of the material by causing a degree of sintering which improves the unity of the briquettes particles. Optimal moisture levels are one of the focuses of current experimental research.

\subsubsection{Size of the Input Fraction}

The size of the input fraction also has an important effect on the densification process because larger input fractions increase the energy needed for densification. However, briquettes formed from large input fractions have lower homogeneity and strength. On the other hand, a large portion of fine particles allows for better material densification. The resulting briquette is uniform, of high quality, and reaches higher volumetric density. With increasing fraction size, the bond strength between particles decreases, causing them to crumble. The quality of a briquette, as a result, decreases and the necessary pressing pressure increases. When pressing, especially 
without additives, the surfaces of the grains must contact on the greatest possible area. The size of this contacting area increases as the fraction size becomes finer and higher pressing pressures are applied. Existing relationships prove that the size of the input fractions should be as small as possible in order to obtain the desired briquette strength (Kádarová, 2005).

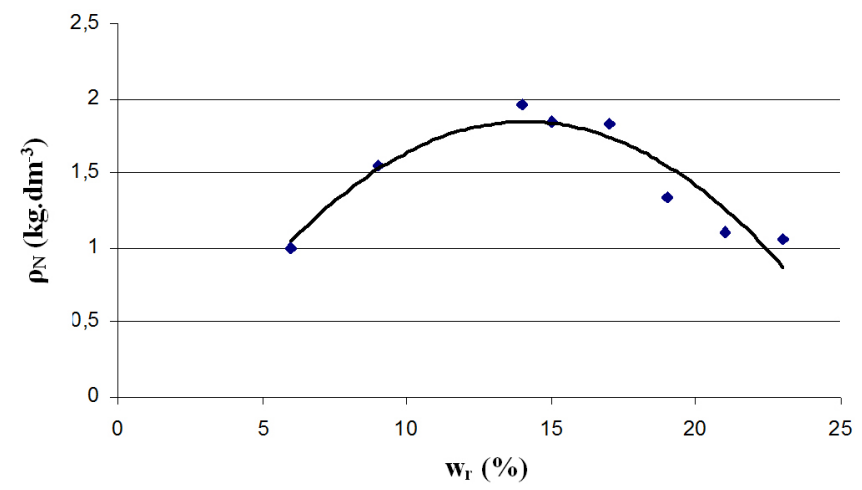

Figure 2.41: Dependence of briquette density on the moisture level of the material (wood sawdust) (Kádarová, 2005).

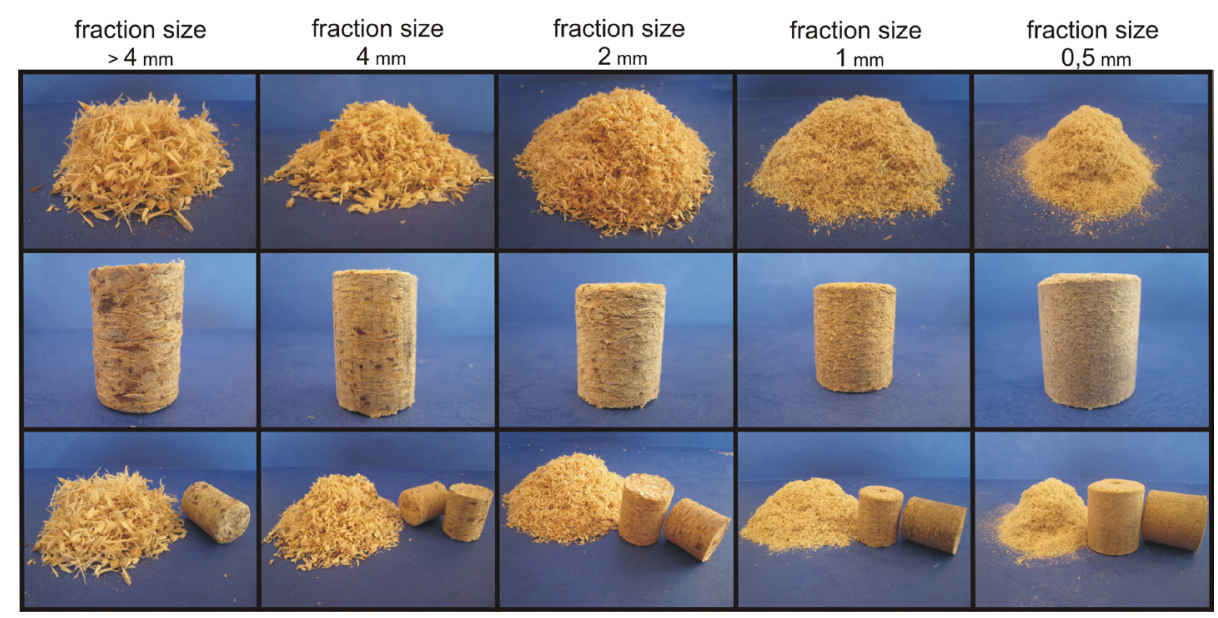

Figure 2.42: Comparison of briquettes made from Pine sawdust with varying fraction sizes. $(p=159$ $\left.\mathrm{MPa}, \mathrm{T}=25^{\circ} \mathrm{C}, \mathrm{w}_{\mathrm{r}}=10 \%\right)($ Križan, 2009). 
Particles exist in the material that, due to external forces, deform and load mainly the contact regions. In terms of densification, it is very important that the described forces are created between particles. The strength of the resultant bonds increases with decreasing fraction size. However, the optimum fraction size changes with respect to the densification technology employed and the raw material used. Therefore, it is important to investigate the effects of fraction size on the resulting density and strength of the briquette. Fraction size is thus one of the parameters that I attempted to identify and verify through the following experiments.

\subsection{Mathematical Model of the Pressing Process of Wood Biomass}

This chapter summarizes the knowledge obtained from various models which best describe uni-axial pressing technology. One of the goals of our experimental research is to identify relationships between pressing wood biomass and the basic theory for pressing. Because one of the stated goals was to create a mathematical model for the pressing process, it was also necessary to perform an analysis of existing mathematical models and compare and evaluate them. It is necessary to understand the pressing process itself - densification, pressure distribution within the chamber, effects of chamber geometry on the pressure distribution, and so on. Based on this knowledge, the effects occurring within the pressing chamber during compaction and the resulting behaviour of the material can be effectively described. The mechanical properties of all wood materials can be improved by the densification of their components (increasing the unit volume of wood). How the force is applied in the pressing process determines whether the process is a uniaxial (single-axis) or multiaxial (hydrostatic) pressing process.

\subsubsection{Theory for Uniaxial Pressing of Particulate Matter}

The boundary conditions for uniaxial pressing are kinematic $\left(\varepsilon_{\mathrm{r}}=0, \varepsilon_{\mathrm{a}}=\varepsilon_{\mathrm{v}}\right)$, and the stress tensor is anisotropic (which is why the process is also known as anisotropic consolidation). An undesirable effect in uniaxial pressing is shell friction, which partially decreases the theoretical value of anisotropic consolidation. Anisotropic stresses in uniaxial pressing creates a compressive structure, and therefore, compressive anisotropy. The nonzero stress deviator evokes dilatational behaviour (Feda, 1977).

According to Feda (1977), uniaxial pressing of a linear, elastic substance under a constant coefficient of lateral pressure $K_{0}$ creates a condition that has been experimentally verified to behave according to Hooke's law:

$$
\varepsilon_{\alpha}=\frac{1-2}{E_{v}}\left(1+2 K_{0}\right) \sigma_{\alpha}(-)
$$


where $E_{v}$ is the module of elasticity in the vertical direction. For elastic deformation, the structure does not change, and therefore $E_{v}=k o n s ̌ t$. Deformation of the particle matter occurs primarily due to irreversible shifting of the particles. Because the structure of the particle matter changes, $E_{v} \neq k o n s t$ and the stress function $\sigma_{a}$, which characterizes the intensity of the structural alteration, also changes. Equation 2.19 can then be written:

$$
d\left(\frac{d h}{h_{0}}\right)=\frac{d \sigma}{E_{0}}(-)
$$

where $E_{0}$ is the volumetric module of elasticity $(\mathrm{MPa}), \sigma=\left(\sigma_{\mathrm{s}}\right)$ is the average stress (MPa), and $h_{0}$ is the initial height of the sample (mm).

In general, Equation (2.22) is used for uniaxial pressing, where $\sigma=\sigma_{\mathrm{a}}$.

After mathematical revisions and the substitution of the boundary conditions, according to Feda (1977),the final relationship for two basic types of compression curves can be achieved. In cases of linear changes in the module of deformation $E_{0}^{\prime}$ along with the stress $\sigma$ for $n^{\prime}=1$ (exponent describing the intensity of structural changes), the following equation is valid:

$$
\Delta e=C\left(1+e_{0}\right) \ln \frac{\sigma}{\sigma_{0}}(-)
$$

Where $\mathrm{C}$ is the compressibility coefficient, $n^{\prime}=0$, representing an unchanged structure. However, particulate matter has $n^{\prime}>0$. In cases where $n^{\prime} \neq 1$, we can substitute $C\left(1-n^{\prime}\right)=C_{0}$ and $n^{\prime}-1=n$ to obtain:

$$
\Delta e=C_{0}\left(1+e_{0}\right)\left[1-\underset{\sigma}{\left.1-\left(\frac{\sigma_{0}}{)^{n}}\right)^{n}\right](-)}\right.
$$

where $\Delta e=e_{0}-e$ is the change in porosity

and $e_{0}$ is the initial value of porosity for $\sigma=\sigma_{0}$ (Feda, 1977).

In the literature, many analytical expressions are given for the compressibility curve, but these expressions often contradict each other regarding the value of porosity. For $n^{\prime}$ approaching +1, the curves are rather similar. According to Feda (1977), Equation2.23 applies for pressing brittle materials with low plasticity, and Equation 2.24 applies for pressing plastic substances. Furthermore, according to Feda (1977), it is obvious that Equation 2.23 for $n^{\prime}=1$ is more realistic for predicting the slower growth in resistance of the particulate matter against compression, where the sliding contact becomes 
predominant and the loading character significantly changes the structure of the particulate matter. Its range of application is therefore limited to all brittle powders, with a "sliding" factor to describe the contacts grinding and regenerating, including metallic powders with an oxidized surface, mainly at low pressures, and other substances with anisotropic particles, such as textile materials, wood, saw dust, and shavings, etc.. For all of these raw materials, the contacting bonds do not significantly grow under pressure, and a highly compressive structure results. Equation 2.24 for $n<0$ characterizes a higher growth in structural resistance to compression caused by a gradual increase in contacting pairs. Its validity is therefore considered mainly for powdery soft metals; metals subject to higher pressures, where the surface oxide is breached and the coefficient of intergranular friction sharply increases; and for particulate matter with isometric grains.

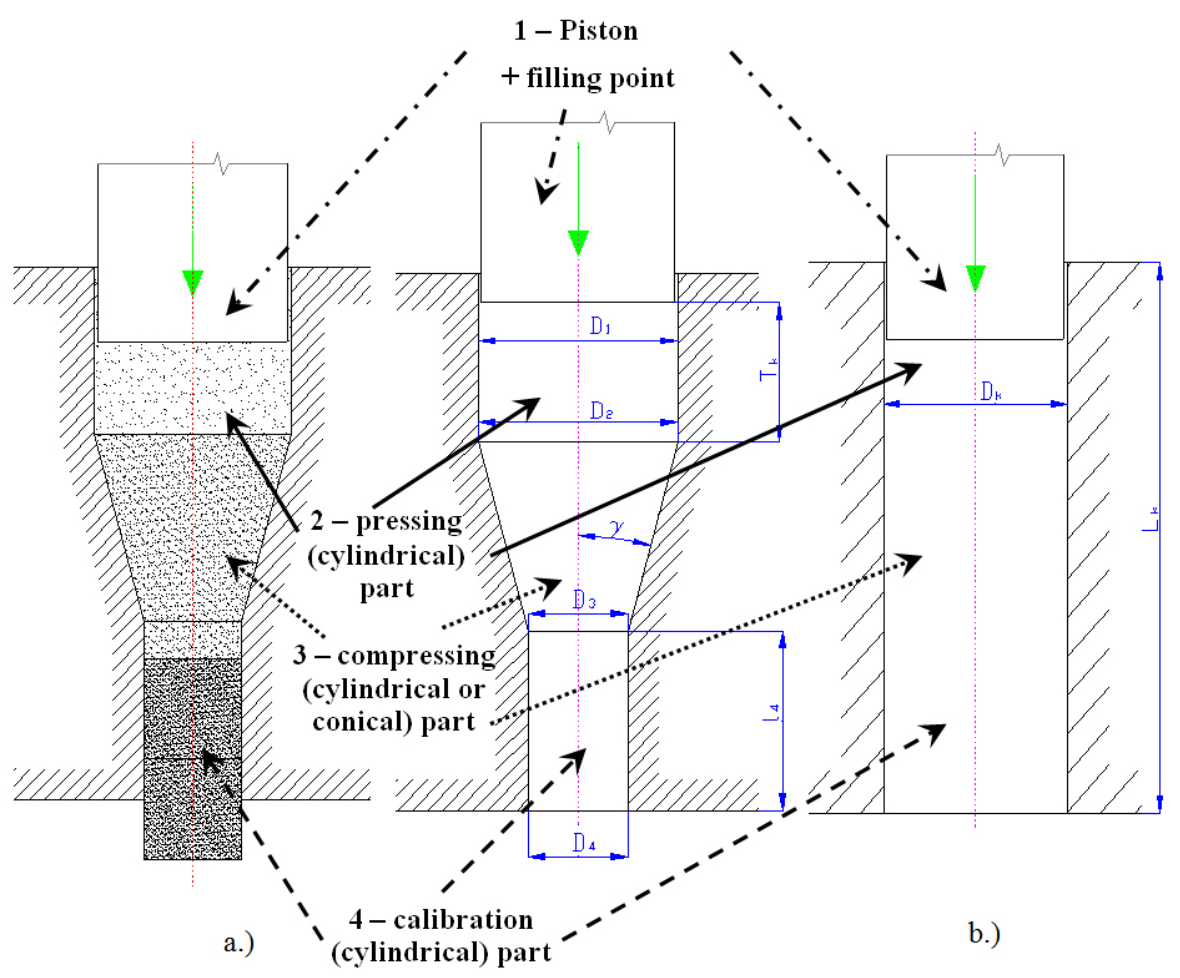

Figure 2.43: Basic parts and main dimensions of the pressing chamber a.)conical; b.) cylindrical (Storožev\& Popov, 1978). 


\subsubsection{Theory of Forward Pressing}

The description of uniaxial pressing requires the knowledge and description of forward pressing as one of the basic technologies for cold, volumetric forming. When forming by pressing, material pours from a closed cavity through an opening. The shape of this opening defines the lateral cross-section of the briquette. This process can be related to the densification of biomass. All existing principles, even densification technologies, utilize these concepts. The theory of forward pressing and the description of pressure ratios within the pressing chamber are given for the simplest case, where the pressing chamber is constructed of four parts (Fig. 2.43). This type of pressing chamber is most commonly used in the construction of pressing machines. Pressing chambers with cylindrical cross-section are also used (Fig. 2.43) over the entire length of the chamber or a conical chamber over the entire length. Cylindrical or ply matrices, the opening of the briquette machine, use a diverse variation of cylindrical and conical shapes (Fig. 2.44). The described type within this work mimics typically-used pressing chambers.

In the schematic below, the pressing part (2) acts as the input container. Material is transported to this part, and under external forces from the pressing piston (1), the densification process may begin. The (conical) compacting part (3), known also as the redactor, is part of the pressing chamber where the basic deformation of the input material is performed. It is here that the densification process is performed due to pressing forces and the pressure against the chamber walls. In the calibration part (4), the briquette is kept under pressure for a specific period of time to reduce the effects of dilation (Blaščík \& Polák, 1987; Storožev \& Popov, 1978). The calibration part gives the briquette its final shape and holds it for a defined period under pressure and heat to reduce the tendency of the briquette to crumble after leaving the pressing chamber. A briquette pushed through a cylindrical calibration part usually changes very little, and the changes that do occur are a result of dilation and elastic limits of the briquette. The magnitude of their effects depends on pressing parameters (pressing temperature and pressure) and the properties of the pressed material (moisture content and fraction size). Due to dilation, a typical undesirable side effect, a stabilization phase must be ensured throughout which dilation occurs. After this time, the briquette is considered to be stable and its density can be determined. Throughout dilation, and more so if excess moisture is present, the briquette changes dimensions and weight. Within the briquette, cracks occur, which reduce the density and quality of the briquette. Cracking also affects the resistance to crumbling and the moisture saturation from the air. The briquette can crumble when manipulated. The stabilization time (dilation time) can be shortened by using a longer calibration part in the chamber and employing the optimum levels of moisture and fraction size in the input material. 


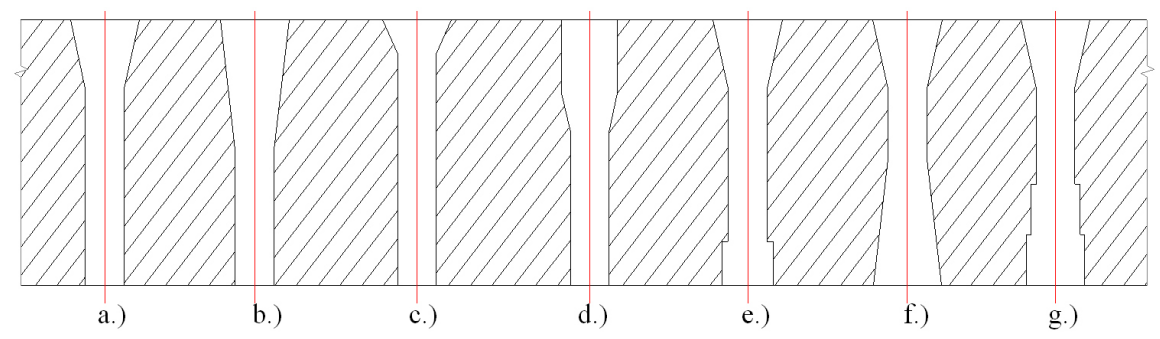

Figure 2.44: Examples of different chamber cross sections - a.)classic; b.) deep; c.) shallow; d.) well; e.) cylindrical; f.) conical; g.) step (Perez, 2009).

The pressure ratios are calculated separately for parts 2, 3, and 4 of the pressing chamber (Storožev \& Popov, 1978). The resulting relationship for calculating the forming (pressing) pressure within the chamber is given as a sum of the pressing pressures acting within each part of the chamber. The following formulas are valid for pressing chambers which have a conical pressing part. Describing the pressure ratios within the pressing chamber based on the fundamental theory for forward pressing can be explained for part 4 of the chamber. The required pressure required in a cylindrical cross section of the calibration part is given by (Storožev\& Popov, 1978):

$$
p_{4}=\sigma_{k} \frac{4 \cdot \mu \cdot l}{D_{4}}(\mathrm{MPa})
$$

where: $\mu$ - friction coefficient $(-) ; \sigma_{k}$ - yield strength of the material (MPa); $\mathrm{D}_{4}-$ diameter of the calibration part of the pressing chamber $(\mathrm{mm}) ; l_{4}-$ length of the calibration part (mm).

Calculating the pressure distribution in the pressing part, conical part 3, is described by equation 2.26 (Storožev \& Popov, 1978). This equation, however, is valid for pressing chambers with a small angle $y$.

$$
p_{3}=\sigma_{k} \cdot\left(1+\frac{\mu+0,5}{2 \cdot \gamma}\right) \cdot \ln \frac{S_{2}}{S_{4}}+p_{4}(\mathrm{MPa})
$$

where: $y$ - conicity of the walls in part $3\left(^{\circ}\right)$

$\mathrm{S}_{2}$ - cross section area of the output of the pressing part $\left(\mathrm{mm}^{2}\right)$

$S_{4}$ - cross section area of the input into the calibration part $\left(\mathrm{mm}^{2}\right)$ 


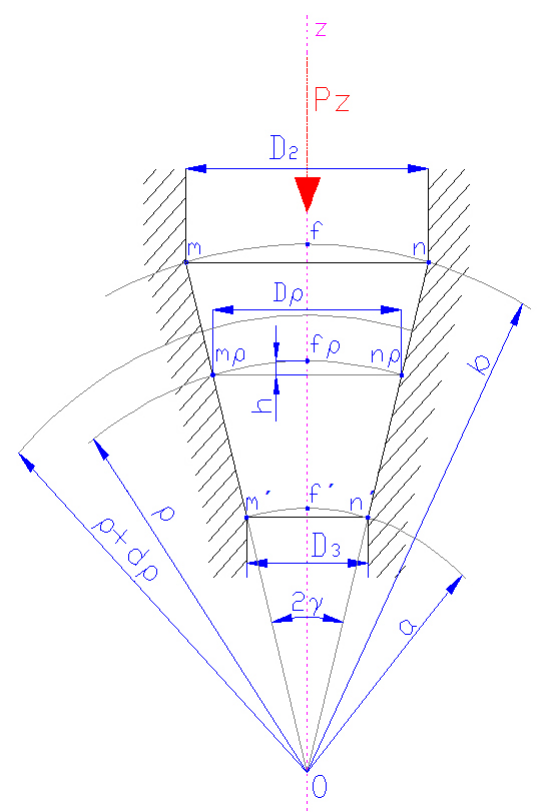

Figure 2.45: Spherical coordinates describing the upper and lower interfaces of plastic deformation concentration in a conical pressing chamber (Storožev \& Popov, 1978).

When investigating the conical pressing part (3), spherical coordinates are used (Storožev \& Popov, 1978). The interface between the concentrated plastic deformation approximates the area $m f n$ of the spherical shelter with the radius $b$ and limit angle $2 y$ (Fig. 2.45); in this case, $f$ represents concentration and not the friction coefficient. The lower interface is represented by the area $m^{\prime} f^{\prime} n^{\prime}$ of the spherical shelter with a radius $a$ and the same limit angle $2 y$. In this case the diameter $D_{3}$ is equal to $D_{4}$. The authors of a well-established study (Storožev \& Popov, 1978) assume that the particle shift at the concentration of deformation occurs on the radius $\rho$. Determining $d V$, they take an elementary volume, bordered by the spherical surfaces with a radius satisfying the values of $\rho$ and $\rho+d \rho$ and conical chamber surface.

The plastic deformation concentration of our material features a large inconsistency between the grain size and the occurrence of residual stresses. In practice, the predominant first type of deformation concentration in the pressing part (2) originates during pressing (2). Because the plastic deformation in the pressing part (2) in this case practically doesn't exist, we can assume that the material is shifted as a whole and has equally distributed elastic pressure. Storožev\& Popov(1978) show that, in favourable friction conditions, the pressures on the walls of the feeding chamber are much lower than the axial pressure. Friction forces decrease linearly with shorter stroke lengths of the piston $T_{k}$. These problems are solved by means of the balanced work method (Storožev\& Popov, 1978). The fundamental law of this method is, for 
plastic deformation, that the work of external forces acting on specific paths is equal to the work of the internal forces $\left(A_{\text {out. }}=A_{\text {int. }}\right.$ ). For this case, work $A_{3}$ was set to be equivalent to the sum of the work of the resulting force $F_{3}$ of the active pressure on the upper interface where the plastic deformation is concentrated plus the total deformation work to change the shape $\left(A_{D}\right)$ plus the work of the contact friction forces on the conical surface $\left(A_{f}\right)$ and the work needed to overcome the resistance in the calibration part (4).

The pressure on the pressing piston acting in part 2 of the chamber is calculated by the following equation (Storožev \& Popov, 1978):

$$
p_{2}=p_{3}+\sigma_{k} \cdot \frac{2 \cdot T_{k}}{D_{2}}(\mathrm{MPa})
$$

where: $T_{k}$ - distance between the piston and lower end of the pressing part of the chamber $(\mathrm{mm}) ; D_{2}-$ is the diameter of the pressing part $(\mathrm{mm})$

The resulting relationship for the calculation for (pressing) pressure in the pressing chamber is determined by the following relation, where we substitute into Equation 2.27 the value of $p_{3}$ from Equation 2.26 and $p_{4}$ from Equation 2.25(Storožev \& Popov, 1978):

$$
p=\sigma_{k} \cdot\left(\ln \frac{S_{2}}{S_{4}}+\ln \frac{S_{2}}{S_{4}} \cdot \frac{\mu+0,5}{2 \cdot \gamma}+\frac{2 \cdot T_{k}}{D_{2}}+\frac{4 \cdot \mu \cdot l_{4}}{D_{4}}\right)(\mathrm{MPa})
$$

This simple procedure allows for the design of an appropriate, optimal shape for the pressing chamber. It also allows for the calculation of acting pressures in the pressing chamber.

\subsubsection{Mathematical Model - "Force Relations in the Channel"}

The mathematical model in this section describes the pressure ratios within an enclosed pressing chamber of a uniaxial vertical press. The use of a backpressure plug prevents material loss when filling the chamber (Fig. 2.46). The pressure ratio within the pressing chamber between the piston and plug are explained by Horrighs (1985) for an element of pressed material $d x$ with neglected briquette weight throughout the pressing process.

From the pressure ratios within the chamber, it is possible to express the equation for force equilibrium as: 


$$
\left[p_{m}-\left(p_{m}+d p_{m}\right)\right] \frac{\pi D_{k}^{2}}{4}-\mu p_{r} \pi D_{k} d x=0
$$

When pressing bulk materials, we typically deal with anisotropic pressure (higher pressures are perpendicular). The ratio between the primary stresses (radial $\sigma_{r} /$ axial $\sigma_{m}$ ) is defined as the residual pressure coefficient, or horizontal pressing ratio $\lambda$. For a disperse material, $\lambda$ reaches values from 0 to 1 .

$$
\lambda=\frac{\sigma_{r}}{\sigma_{m}}=\frac{p_{r}}{p_{m}} \Rightarrow p_{r}=\lambda p_{m}(\mathrm{MPa})
$$

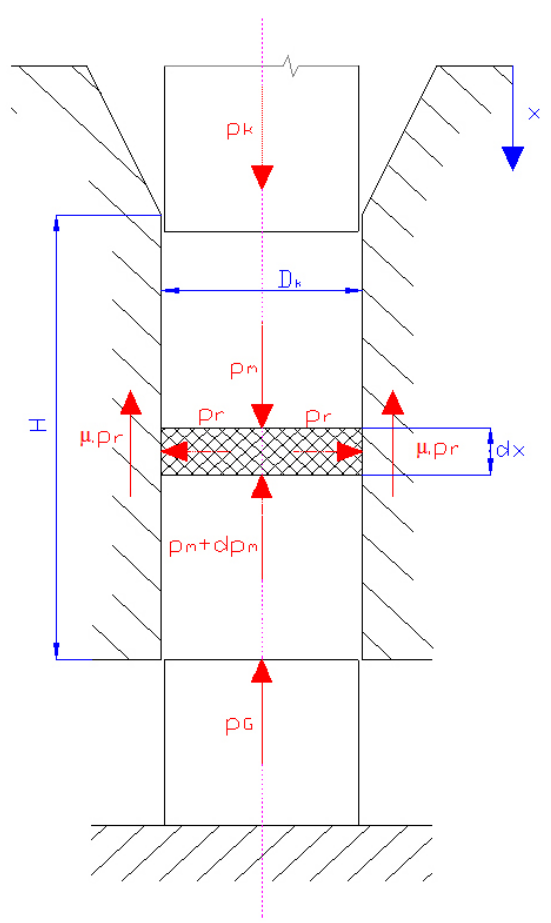

$p_{k}$ - axial piston pressure ( $\left.\mathrm{MPa}\right)$

$p_{G}$ - backpressure in the press housing ( $\left.\mathrm{MPa}\right)$

$p_{r}$ - radial pressure ( $\mathrm{MPa}$ )

$p_{m}$ - axial pressure on the plug ( $\mathrm{MPa}$ )

$D_{k}$ - diameter of the pressing chamber $(\mathrm{mm})$

$\mu$ - coefficient of bond friction (-)

$\mathrm{H}$ - length of the compressed plug ( $\mathrm{mm}$ )

Figure 2.46: Pressure distribution in the pressing chamber in a uniaxial vertical press (Horrighs, 1985).

After substituting Equation 2.30 into Equation 2.29, and applying the following boundary conditions: $x=0, p_{m}=p_{k}$, pre $x=H, p_{m}=p_{G}$

The following relation is obtained (Horrighs, 1985):

$$
p_{k}=p_{G} \cdot e^{\frac{4 \cdot \lambda \cdot \mu \cdot H}{D_{k}}(\mathrm{MPa})}
$$


Equation 2.31 gives the relationship between the piston pressure $p_{k}$ and the backpressure acting on the pressed material $p_{G}$. This relationship can be used for pressing in an open chamber where the back pressure is generated by static friction between the material and chamber walls. From this relation, we can further determine the magnitude of necessary backpressure in the operation and determine the static friction in the press column (Feda, 1977). Modifying Equation 2.31 can yield values for $p_{G}$ for any desired value of $p_{k}$.

$$
p_{G}=p_{k} \cdot e^{-\frac{4 \cdot \lambda \cdot \mu \cdot H}{D_{k}}(\mathrm{MPa})}
$$

Experience has shown that, after pressing, it is still necessary to determine the pressure of the pressed plug. Horrighs (1985) developed a formulation of relationships for the pressed plug because, after freeing the load on it, residual radial pressure dominates within the plug $\left(p_{r, r}\right)$. This residual pressure causes briquettes to fracture. Therefore, it is very important to determine the relationship between the pressing pressure and length of the chamber (calibration part). Throughout the pressing process, the axial stress is dominant. After pushing out the plug, the dominant stress becomes radial. This means that the change in direction of stress must occur under the unloaded state - when pushing out the plug. Therefore, a point exists when the axial and radial stress are equal (hydrostatic stress state). After this point, the radial stresses become larger than the axial stresses until pressure is completely removed and residues of axial stress remain. The briquette is necessary to push out of the pressing chamber only when its strength $\mathrm{f}_{\mathrm{c}}$ is greater than the residual radial stresses $\sigma_{r, r}$, or $p_{r, r}$. If this condition is satisfied, it is ok to push the briquette out of the calibration part of the pressing chamber.

The behaviour of the axial and radial pressure is formulated with respect to the initial compaction in the pressing phase with relief and over-loading states in the pressing phase according to the following relations (Horrighs, 1985):

a) for pressing the plug (plastic range), the following is true:

$$
p_{r}=\lambda \cdot p_{m}(\mathrm{MPa})
$$

b) for the movement of the already compacted plug (elastic range), the following is true:

$$
p_{r}=A \cdot p_{m}+p_{r, r}(\mathrm{MPa})
$$

Where the constant A represents the material constant, which gives the degree of elastic properties in the pressed material. 


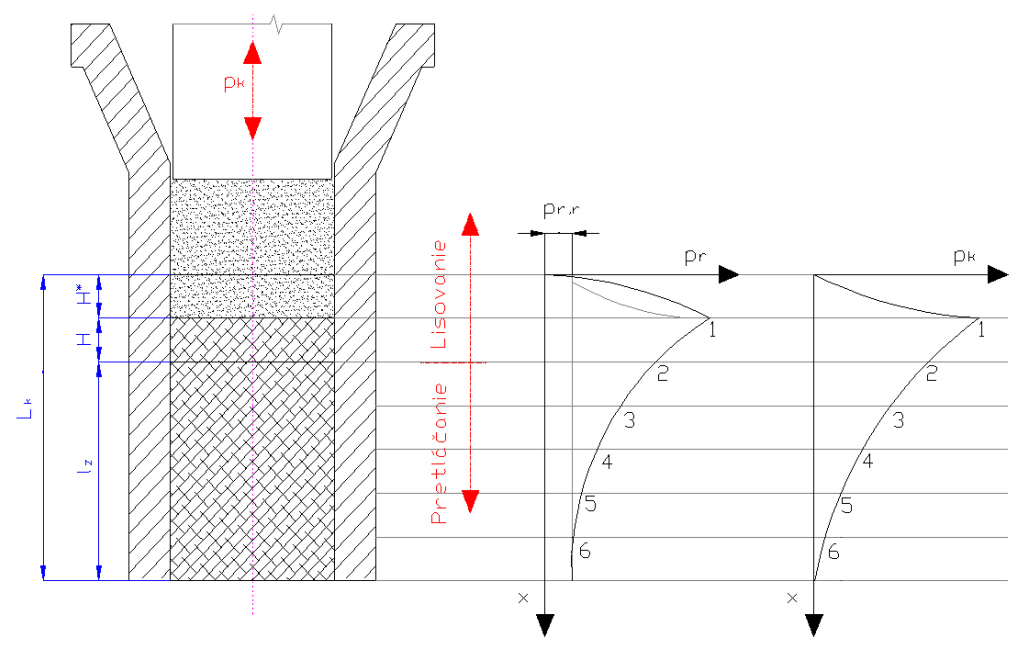

Figure 2.47: Behaviour of the radial and axial pressure when pressing in the pressing chamber (Horrighs, 1985).

Based on pressure conditions in the pushing phase, force equilibrium at any random point (of the already pressed material) of an element (Fig. 2.46) that is part of the pressing column (Fig. 2.47) can be evaluated. With the applied boundary conditions for $x=l_{z}$, and considering Equation 2.34 Horrighs, 1985), we can formulate a relationship (2.35) between the pressure on the evenly compressed plug and the length of column $l_{z}$ :

$$
p_{m}(x)=\frac{p_{r, r}}{A} \cdot\left(e^{\frac{4 \mu \cdot A}{D_{k}} \cdot(1-x)}-1\right)(\mathrm{MPa})
$$

After substituting Equation 2.32 into Equation 2.35, we obtain the following relation:

$$
l_{z}=\frac{D_{k}}{4 \cdot \mu \cdot A} \cdot \ln \left(A \cdot \frac{p_{G}}{p_{r, r}}+1\right)(\mathrm{mm})
$$

The overall length of the pressing channel $L_{k}$ is given by the sum of column length $l_{z}$ and plug length $H ; L_{k}$ can be calculated directly from the following Equation 2.37, since $l_{z}>>H$ : 


$$
L_{k}=\frac{D_{k}}{4 \cdot \mu \cdot A} \cdot \ln \left(A \cdot \frac{p_{G}}{p_{r, r}}+1\right)(\mathrm{mm})
$$

Calculating the length of the pressing channel (length of the pressed column) is possible only with known length of the pressed plug $H$ (using the pressing ratio, dimensions of the pressing chamber, and the material properties) for known pressure $p_{k}$ and known material constants $\mu, \lambda, A$, a, and $p_{r, r^{\circ}}$

\subsubsection{Mathematical Model - "Pure Elastic Case“}

The next mathematical model, stated according to Holm \& et al.(2006), considers the pressing of the elastic material in the pressing chamber. The derived relations give the expressions for changing plug pressure.

The chosen element has the form of a plane disk with thickness $d x$ and radius $r$. The center of this disk is identical with the axis of the press opening. The differential change in pressure $d p_{m}$ through $d x$ is given by the relation:

$$
d p_{m}=\frac{d F_{m}}{\pi \cdot r^{2}} \Rightarrow d F_{m}=d p_{m} \cdot \pi \cdot r^{2}
$$

Friction force $d F \mu$ acting on the differential area 2. $\pi . r d x$ is given by relation:

$$
d F_{\mu}=\mu \cdot d F_{N}=\mu \cdot p_{N} \cdot 2 \cdot \pi \cdot r \cdot d x
$$

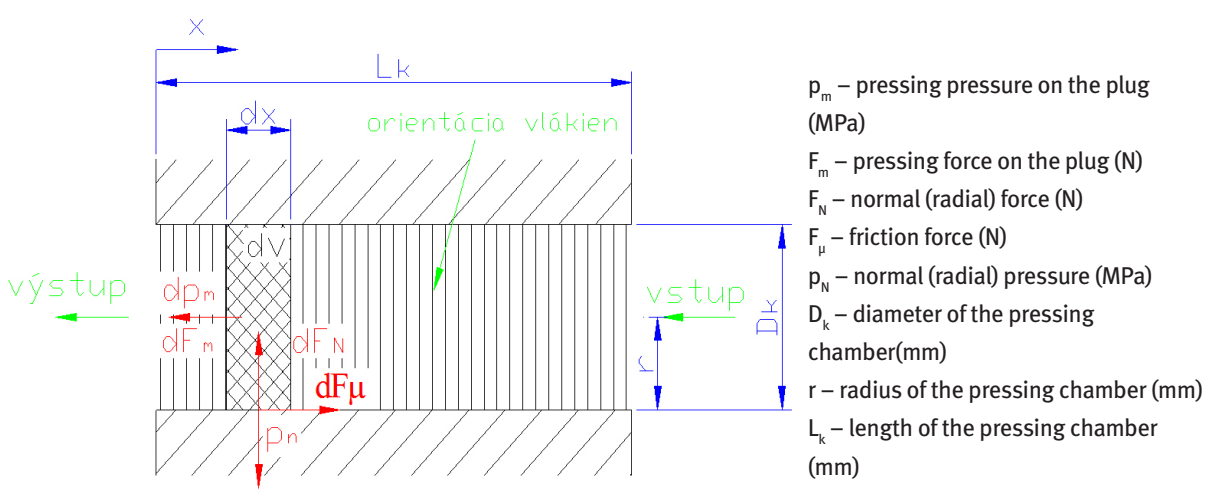

Figure 2.48: Schematic sketch of the forces and pressures acting on an element volume $\mathrm{dV}$ within the pressing channel (Holm \& et al., 2006). 
The following relations are based on the assumption that wood is an orthotropic material (independent mechanical properties in the parallel and perpendicular directions of the grain fibres). Holm \& et al.(2006) assume that the fibre bundles in pellets are oriented perpendicular to the larger dimension of the pressing chamber. Furthermore, there is a consideration that the material has elastic properties (dependence between stress and strain) that are described by Hooke's law. For materials fulfilling Hooke's law, the ratio between stress and strain is constant.

Stress causing expansion is considered positive, whereas stress causing shrinkage is considered negative. If expansion or shrinkage of an element occurs due to some applied force and is lost due to the loss of this force, the material is known as elastic. The relationship between the parallel and perpendicular fibre bundles and radial deformation describes the individual coefficient between materials (Poisson's coefficient). Poisson's coefficient is defined as the ratio of elongation for a specific element $v_{R L}$. Therefore the coefficient $v_{R L}$ can be written as:

$$
v_{R L}=\frac{\text { longitudinal_extension }}{\text { radial_extension }}=\frac{\frac{d v}{d r}}{\frac{d u}{d x}}
$$

The above expression assumes that the material is homogeneous throughout its cross section (Holm \& et al., 2006). In the case of spatial restrictions of pellets in the pressing channel, radial expansion is not possible, and therefore elongation manifests itself as a stress on the walls of the pressing channel. Hooke's law for the lateral and radial directions of fibre bundles can be written as follows:

$$
\begin{aligned}
& F_{L}=E_{L} \cdot S_{r} \cdot \frac{\Delta r}{r}(\mathrm{~N}) \\
& F_{R}=E_{R} \cdot S_{k} \cdot \frac{d u}{d x}(\mathrm{~N})
\end{aligned}
$$

where $E_{L}$ and $E_{R}$ are modules of elasticity in the lateral and radial directions of the fibre bundles, respectively. $S_{r}=2 . \pi . r . d x$ is the area of the element side shell, and $S_{k}=\pi \cdot r^{2}$ is the cross-sectional area of the element. Furthermore, if the acting pressure in the lateral and radial $\left(p_{r} / p_{k}\right)$ directions are compared and the following conditions are applied (Holm \& et al., 2006): $p_{r}$ is equal to $p_{N}$ and $p_{k}$ is equal to $p_{m}$ Then:

$$
p_{N}=G \cdot \cup_{R L} \cdot p_{m}(\mathrm{MPa})
$$

where $G$ is the ratio between the module of elasticity in the lateral and radial directions of the fibre bundles $\left(E_{L} / E_{R}\right)$. 
If the following equations (2.38 and 2.39) are made equal to each other, we obtain the differential equation in $p_{m}$ :

$$
d F_{m}=d F_{\mu} \rightarrow d p_{m} \cdot \pi \cdot r^{2}=\mu \cdot p_{N} \cdot 2 \cdot \pi \cdot r \cdot d x
$$

After rewriting these equations using Equation 2.43 and its solution for $x-x_{0}=L_{k}$ according to [11], we obtain the resulting relation, which gives the relation for the change in pressing pressure on the plug in the pressing channel of the chamber, where $p_{m 0}$ is the initial pressing pressure:

$$
p_{m}=p_{m o} \cdot e^{\frac{2 . \mu \cdot G \cdot v_{R L} \cdot L_{k}}{r}}(\mathrm{MPa})
$$

\subsubsection{Mathematical-physical Model - "Material Composition"}

The following mathematical-physical model is a model which best reflects the target model of experimental research. It was designed by Kádarová (2005). It is shown that the densification process itself is very complicated and involves many parameters affecting its process. This mathematical model is valid only within a certain range of pressure changes and temperature intervals of the material. The mathematicalphysical model designed and based on experimental results has the following form (Kádarová, 2005):

$$
\rho=A_{K} \cdot(p+B) \cdot e^{k p \cdot\left(p_{\max }-p\right)}
$$

where: $\rho$ - is the resulting density $\left(\mathrm{kg} \cdot \mathrm{dm}^{-3}\right) ; A_{K}$ - coefficient calculated based on experimental results characterising shape and curvature of the curve; $B$ - material constant; $k p$ - coefficient related to the pressure, calculated from experimental results; $p_{\max }$ - coefficient chosen based on monitoring of pressure (maximum pressure) (MPa); $p$ - pressing pressure $(\mathrm{MPa})$

This equation characterises the relationship between briquette density and pressing pressure. For every temperature level, it was necessary to calculate from experimental values. Coefficient $k p$ was calculated based on experimental results. Increasing the densification pressure allows us to reach high values of volumetric density. Based on monitored pressures (Kádarová, 2005), the maximum effective pressing pressure was determined to be $p_{\max }=400 \mathrm{MPa}$. It is irrational and, in most cases, ineffective to exceed this pressure value. Then, Equation 2.46 has the following form:

$$
\rho=A_{K} \cdot(p+B) \cdot e^{k p \cdot(400-p)}
$$


Experiments were performed with the following materials - spruce and oak sawdust, straw, and stems (Kádarová, 2005). In this work, the following material constants (B) were experimentally obtained:

- $\quad$ spruce sawdust, $\mathrm{B}=96$

- oak sawdust, $\mathrm{B}=110$

- straw, $B=106$

- stems, $\mathrm{B}=221$

Constant B depends on the content of volatile substances up to $76 \%$, where it sharply decreases (Kádarová, 2005). A similar behaviour can be related to cellulose content up to $48 \%$. Increasing these variables has little effect on the constants. Based on this work, the author concludes that, within the range of these changes and for the content of volatile substances and cellulose, constant $B$ results as the material constant.

Similarly, the experimental findings and values for coefficient $A_{K}$ for each mentioned material are reported (Kádarová, 2005). However, this coefficient changes with respect to temperature, meaning that it is a function of the material temperature. Constant $k p$ is a coefficient related to the pressure and is the same as coefficient $A_{K}$ for the functional temperature of the material

\subsubsection{Summary}

From the above mathematical descriptions, one can understand that not many mathematical models describing the densification process exist. The ones that do exist are based on pressing theory and describe the pressure ratio throughout the pressing process. These models can be used in the design of the pressing chamber or the formulation of a mathematical model that describes the effects of structural parameters on the resulting briquette quality. However, models describing the effects of changing technological parameters on the pressing process and effects on the pressed material do not exist. It is very important to find a relationship between the density of the briquette and pressing pressure, temperature, moisture content, and input fraction size. Knowledge of these relationships could provide an excellent insight for designing pressing machines (chambers). The experiments within this monograph hence strive to design and justify the use of a mathematical model describing the densification process for application to real practices. 